\title{
Toward Neural-Network-Guided Program Synthesis and Verification *
}

\author{
Naoki Kobayashi ${ }^{1}$ (D), Taro Sekiyama ${ }^{2}$ (D) Issei Sato ${ }^{1}$, and Hiroshi Unno ${ }^{3,4}{ }^{(\mathbb{C}}$ \\ 1 The University of Tokyo, Tokyo, Japan, \{koba,issei.sato\}@is.s.u-tokyo.ac.jp \\ 2 National Institute of Informatics \& SOKENDAI, Tokyo, Japan, \\ tsekiyama@acm.org \\ 3 University of Tsukuba, Ibaraki, Japan \\ 4 RIKEN AIP, Tokyo, Japan
}

\begin{abstract}
We propose a novel framework of program and invariant synthesis called neural network-guided synthesis. We first show that, by suitably designing and training neural networks, we can extract logical formulas over integers from the weights and biases of the trained neural networks. Based on the idea, we have implemented a tool to synthesize formulas from positive/negative examples and implication constraints, and obtained promising experimental results. We also discuss two applications of our synthesis method. One is the use of our tool for qualifier discovery in the framework of ICE-learning-based CHC solving, which can in turn be applied to program verification and inductive invariant synthesis. Another application is to a new program development framework called oracle-based programming, which is a neural-network-guided variation of Solar-Lezama's program synthesis by sketching.
\end{abstract}

\section{Introduction}

With the recent advance of machine learning techniques, there have been a lot of interests in applying them to program synthesis and verification. Garg et al. [6] have proposed the ICE-framework, where the classical supervised learning based on positive and negative examples been extended to deal with "implication constraints" to infer inductive invariants. Zhu et al. [32] proposed a novel approach to combining neural networks (NNs) and traditional software, where a NN controller is synthesized first, and then an ordinary program that imitates the NN's behavior; the latter is used as a shield for the neural net controller and the shield (instead of the NN) is verified by using traditional program verification techniques. There have also been various approaches to directly verifying NN components $[8,30,1,21,16]$.

We propose yet another approach to using neural networks for program verification and synthesis. Unlike the previous approaches where neural networks are used either as black boxes $[32,28]$ or white boxes $[8,11]$, our approach treats

* An Extended Version of the Summary in the Proceedings of SAS 2021, Springer LNCS. 


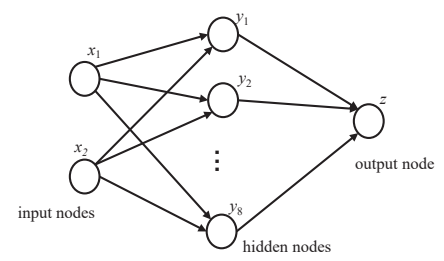

Fig. 1. A Neural Network with One Hidden Layer

neural networks as gray boxes. Given training data, which typically consist of input/output examples for a (quantifier-free) logical formula (as a part of a program component or a program invariant) to be synthesized, we first train a NN. We then synthesize a logical formula by using the weights and biases of the trained NN as hints. Extracting simple (or, "interpretable"), classical ${ }^{5}$ program expressions from NNs has been considered difficult, especially for deep NNs; in fact, achieving "explainable AI" [2] has been a grand challenge in the field of machine learning. Our thesis here is, however, that if $\mathrm{NNs}$ are suitably designed with program or invariant synthesis in mind, and if the domain of the synthesis problems is suitably restricted to those which have reasonably simple program expressions as solutions, then it is actually often possible to extract program expressions (or logical formulas) by inspecting the weights of trained NNs.

To clarify our approach, we give an example of the extraction. Let us consider the three-layer neural network shown in Figure 1. The NN is supposed to work as a binary classifier for two-dimensional data: it takes a pair of numbers $\left(x_{1}, x_{2}\right)$ as an input, and outputs a single number $z$, which is expected to be a value close to either 1 or 0 . The NN has eight hidden nodes, and the sigmoid function $\sigma$ is used as activation functions for both the hidden and output nodes. ${ }^{6}$ The lefthand side of Table 1 shows a training data set, where each row consists of inputs $\left(x_{1}, x_{2}\right)\left(-25 \leq x_{1}, x_{2} \leq 25\right)$, and their labels, which are 1 if $4 x_{1}+x_{2}>$ $0 \wedge 2 x_{1}+3 x_{2}+9<0$. The righthand side of Table 1 shows the weights and biases of the trained NN. The $i$-th row shows information about each hidden node: $w_{1, i}, w_{2, i}$, and $b_{i}(i \in\{1, \ldots, 8\})$ are the weights and the bias for the links connecting the two input nodes and the $i$-th hidden node, and $w_{o, i}$ is the weight for the link connecting the hidden node and the output node. (Thus, the value $y_{i}$ of the $i$-th hidden node for inputs $\left(x_{1}, x_{2}\right)$ is $y_{i}=\sigma\left(b_{i}+w_{1, i} x_{1}+w_{2, i} x_{2}\right)$, and the output of the whole network is $\sigma\left(b_{o}+\sum_{i=1}^{8} w_{o, i} y_{i}\right)$ for some bias $b_{o}$.) The rows are sorted according to the absolute value of $w_{o, i}$. We can observe that the ratios among $w_{1}, w_{2}, b$ of the first four rows are roughly $2: 3: 9$, and those of the last four rows are roughly $4: 1: 0$. Thus, the value of each hidden node is close to $\sigma\left(\beta\left(2 x_{1}+3 x_{2}+9\right)\right)$ or $\sigma\left(\beta\left(4 x_{1}+x_{2}\right)\right)$ for some $\beta$. Due to the property

\footnotetext{
${ }^{5}$ Since neural networks can also be expressed as programs, we call ordinary programs written without using neural networks classical, to distinguish them from programs containing NNs.

6 These design details can affect the efficacy of our program expression extraction, as discussed later.
} 
Table 1. Training Data (left) and the Result of Learning (right)

\begin{tabular}{|c|c|c|c|c|c|c|}
\hline \multirow{2}{*}{$\begin{array}{l}x_{1} \\
25\end{array}$} & \multirow{2}{*}{$\begin{array}{l}x_{2} \\
25\end{array}$} & label & \multirow[b]{2}{*}{$w_{1, i}$} & \multirow[b]{2}{*}{$w_{2, i}$} & \multirow[b]{2}{*}{$b_{i}$} & \multirow[b]{2}{*}{$w_{o, i}$} \\
\hline & & 0 & & & & \\
\hline$\cdots$ & & & 4.037725448 & 6.056035518 & 18.18252372 & -11.76355457 \\
\hline 25 & -21 & 0 & 4.185569763 & 6.27788019 & 18.92045211 & -11.36994552 \\
\hline 25 & -20 & 1 & 3.775603055 & 5.662680149 & 16.86475944 & -10.83486366 \\
\hline 25 & -21 & 1 & 3.928676843 & 5.892404079 & 17.63601112 & -10.78136634 \\
\hline$\cdots$ & & & -15.02299022 & -3.758415699 & 1.357473373 & -9.199707984 \\
\hline 25 & -25 & 1 & -13.6469354 & -3.414942979 & 1.145593643 & -8.159229278 \\
\hline 24 & 25 & 0 & -11.69845199 & -2.927870512 & 0.8412334322 & -7.779587745 \\
\hline$\cdots$ & & & -12.65479946 & -3.168056249 & 0.9739738106 & -6.938682556 \\
\hline-25 & -25 & 0 & & & & \\
\hline
\end{tabular}

that the value of the sigmoid function $\sigma(u)$ is close to 0 or 1 except near $u=0$, we can guess that $2 x_{1}+3 x_{2}+9 \diamond 0$ and $4 x_{1}+x_{2} \diamond 0$ (where $\diamond \in\{<,>, \leq, \geq\}$ ) are relevant to the classification. Once the relevant atomic formulas are obtained, we can easily find the correct classifier $4 x_{1}+x_{2}>0 \wedge 2 x_{1}+3 x_{2}+9<0$ by solving the problem of Boolean function synthesis in a classical manner.

We envision two kinds of applications of our NN-guided predicate synthesis sketched above. One is to an ICE-based learning of inductive program invariants $[6,4]$. One of the main bottlenecks of the ICE-based learning method (as in many other verification methods) has been the discovery of appropriate qualifiers (i.e. atomic predicates that constitute invariants). Our NN-guided predicate synthesis can be used to find qualifiers, by which reducing the bottleneck. The other potential application is to a new program development framework called oraclebased programming. It is a neural-network-guided variation of Solar-Lezama's program synthesis by sketching [27]. As in the program sketch, a user gives a "sketch" of a program to be synthesized, and a synthesizer tries to find expressions to fill the holes (or oracles in our terminology) in the sketch. By using our method outlined above, we can first prepare a NN for each hole, and train the NN by using data collected from the program sketch and specification. We can then guess an expression for the hole from the weights of the trained NN.

The contributions of this paper are: (i) the NN-guided predicate synthesis method sketched above, (ii) experiments that confirm the effectiveness of the proposed method, and (iii) discussion and preliminary experiments on the potential applications to program verification and synthesis mentioned above.

Our idea of extracting useful information from NNs resembles that of symbolic regression and extrapolation [15,20,23], where domain-specific networks are designed and trained to learn mathematical expressions. Ryan et al. [22,29] recently proposed logical regression to learn SMT formulas and applied it to the discovery of loop invariants. The main differences from those studies are: (i) our approach is hybrid: we use NNs as gray boxes to learn relevant inequalities, and combine it with classical methods for Boolean function learning, and (ii) our learning framework is more general in that it takes into account positive/negative samples, and implication constraints; see Section 5 for more discussion. 
The rest of this paper is structured as follows. Section 2 shows our basic method for synthesizing logical formulas from positive and negative examples, and reports experimental results. Section 3 extends the method to deal with $\mathrm{im}$ plication constraints in the ICE-learning framework [6], and discusses an application to CHC (Constrained Horn Clauses) solving. Section 4 introduces the new framework of oracle-based programming, and shows how our synthesis method can be used as a key building block. Section 5 discusses related work and Section 6 concludes the paper.

\section{Predicate Synthesis from Positive/Negative Examples}

In this section, we propose a method for synthesizing logical formulas on integers from positive and negative examples, and report experimental results. We will extend the method to deal with "implication constraints" [6,4] in Section 3.

\subsection{The Problem Definition and Our Method}

The goal of our synthesis is defined as follows.

Definition 1 (Predicate synthesis problem with $\mathbf{P} / \mathbf{N}$ Examples). The predicate synthesis problem with positive $(P)$ and negative $(N)$ examples (the $P N$ synthesis problem, for short) is, given sets $P, N \subseteq \mathbf{Z}^{k}$ of positive and negative examples (where $\mathbf{Z}$ is the set of integers) such that $P \cap N=\emptyset$, to find a logical formula $\varphi\left(x_{1}, \ldots, x_{k}\right)$ such that $\models \varphi\left(v_{1}, \ldots, v_{k}\right)$ holds for every $\left(v_{1}, \ldots, v_{k}\right) \in P$ and $\models \neg \varphi\left(v_{1}, \ldots, v_{k}\right)$ for every $\left(v_{1}, \ldots, v_{k}\right) \in N$.

For the moment, we assume that formulas are those of linear integer arithmetic (i.e. arbitrary Boolean combinations of linear integer inequalities); some extensions to deal with the modulo operations and polynomial constraints will be discussed later in Section 2.3.

The overall flow of our method is depicted in Figure 2. We first (in the NN training phase) train a $\mathrm{NN}$ on a given set of $\mathrm{P} / \mathrm{N}$ examples, and then (in the qualifier extraction phase) extract linear inequality constraints (which we call "qualifiers") by inspecting the weights and biases of the trained NN, as sketched in Section 1. Finally (in the formula synthesis phase), we construct a Boolean combination of the qualifiers that matches $\mathrm{P} / \mathrm{N}$ examples. Note that the trained $\mathrm{NN}$ is used only for qualifier extraction; in the last phase, we use a classical algorithm for Boolean function learning.

Each phase is described in more detail below.

NN Training. We advocate the use of a four-layer neural network as depicted in Figure 3, where the sigmoid function $\sigma(x)=\frac{1}{1+e^{-x}}$ is used as the activation functions for all the nodes.

We briefly explain the four-layer neural network; those who are familiar with neural networks may skip this paragraph. Let us write $N_{i, j}$ for the $j$-th node 


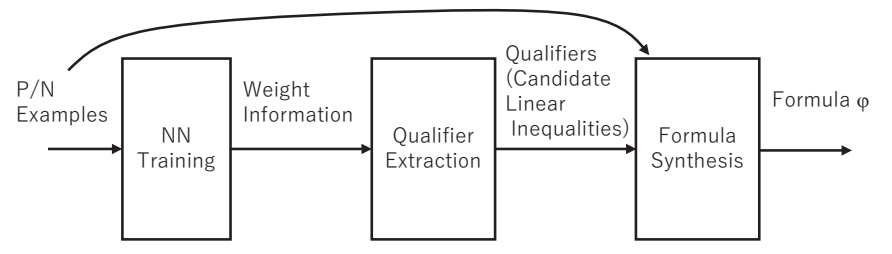

Fig. 2. An Overall Flow of Our Synthesis Framework

in the $i$-th layer, and $m_{i}$ for the number of the nodes in the $i$-th layer (hence, $m_{4}$, which is the number of output nodes, is 1). Each link connecting $N_{i-1, j}$ and $N_{i, k}(i \in\{2,3,4\})$ has a real number called the weight $w_{i, j, k}$, and each node $N_{i, j}$ $(i \in\{2,3,4\})$ also has another real number $b_{i, j}$ called the bias. The value $o_{i, j}$ of each node $N_{i, j}(i>1)$ is calculated from the input values $o_{0, j}$ by the equation:

$$
o_{i, j}=f\left(b_{i, j}+\sum_{k=1}^{m_{i-1}} w_{i, k, j} \cdot o_{i-1, k}\right),
$$

where the function $f$, called an activation function, is the sigmoid function $\sigma$ here; other popular activation functions include $\operatorname{ReLU}(x)=\max (0, x)$ and $\tanh =\frac{e^{x}-e^{-x}}{e^{x}+e^{-x}}$. The weights and biases are updated during the training phase. The training data are a set of pairs $\left(d_{i}, \ell_{i}\right)$ where $d_{i}=\left(o_{1,1}, \ldots, o_{1, m_{1}}\right)$ is an input and $\ell_{i}$ is a label (which is 1 for a positive example and 0 for a negative one). The goal of training a $\mathrm{NN}$ is to adjust the weights and biases to (locally) minimize the discrepancy between the output $o_{4,1}$ of the NN and $\ell_{i}$ for each $d_{i}$. That is usually achieved by defining an appropriate loss function for the training data, and repeatedly updating the weights and biases in a direction to decrease the value of the loss function by using the gradient descent method.

Our intention in choosing the four-layer NN in Figure 3 is to force the NN to recognize qualifiers (i.e., linear inequality constraints) in the second layer (i.e., the first hidden layer), and to recognize an appropriate Boolean combination of them in the third and fourth layers. The sigmoid function was chosen as the activation function of the second layer to make it difficult for the NN to propagate information on the inputs besides information about linear inequalities, so that we can extract linear inequalities only by looking at the weights and biases for the hidden nodes in the second layer. Note that the output of each hidden node in the second layer is of the form:

$$
\sigma\left(b+w_{1} x_{1}+\cdots+w_{k} x_{k}\right) .
$$

Since the output of the sigmoid function is very close to 0 or 1 except around $b+w_{1} x_{1}+\cdots+w_{k} x_{k}=0$ and input data $x_{1}, \ldots, x_{k}$ take discrete values, only information about $b+w_{1} x_{1}+\cdots+w_{k} x_{k}>c$ for small $c$ 's may be propagated 


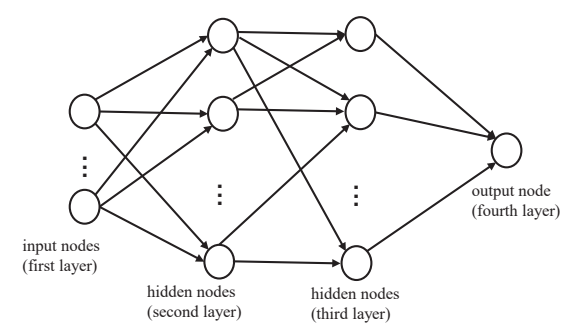

Fig. 3. A Four-Layer Neural Network

to the second layer. In other words, the hidden nodes in the second layer can be expected to recognize "features" of the form $b+w_{1} x_{1}+\cdots+w_{k} x_{k}>c$, just like the initial layers of DNNs for image recognition tend to recognize basic features such as lines. The third and fourth layers are intended to recognize a (positive) Boolean combination of qualifiers. We expect the use of two layers (instead of only one layer) for this task makes it easier for $\mathrm{NN}$ to recognize Boolean formulas in DNF or CNF. ${ }^{7}$ Notice that the conjunction $x_{1} \wedge \cdots \wedge x_{k}$ and the disjunction $x_{1} \vee \cdots \vee x_{k}$ can respectively be approximated by $\sigma\left(\beta\left(x_{1}+\cdots+x_{k}-\frac{2 k-1}{2}\right)\right)$ and $\sigma\left(\beta\left(x_{1}+\cdots+x_{k}-\frac{1}{2}\right)\right)$ for a sufficiently large positive number $\beta$.

For the loss function of the NN, there are various candidates. In the experiments reported later, we tested the mean square error function (the average of $\left(o_{i}-\ell_{i}\right)^{2}$, where $o_{i}$ is the output of NN for the $i$-th training data, and $\ell_{i}$ is the corresponding label) and the mean of a logarithmic error function (the average of $\left.-\log \left(1-\left|o_{i}-\ell_{i}\right|\right)\right) .^{8}$

Qualifier Extraction. From the trained NN, for each hidden node $N_{2, i}$ in the second layer, we extract the bias $b_{2, i}$ (which we write $w_{2,0, i}$ below for technical convenience) and weights $w_{2,1, i}, \ldots, w_{2, k, i}$, and construct integer coefficients $c_{0}, c_{1}, \ldots, c_{k}$ such that the ratio $c_{n} / c_{m}$ of each pair of coefficients is close to $w_{2, n, i} / w_{2, m, i}$. We then generate linear inequalities of the form $c_{0}+c_{1} x_{1}+\cdots+$ $c_{k} x_{k}>e$ where $e \in\{-1,0,1\}$ as qualifiers. The problem of obtaining the integer coefficients $c_{0}, \ldots, c_{k}$ is essentially a Diophantine approximation problem, which can be solved, for example, by using continued fraction expansion. The current implementation uses the following naive, ad hoc method. ${ }^{9}$ We assume that the coefficients of relevant qualifiers are integers smaller than a certain value $K\left(K=5\right.$ in the experiments below). Given $w_{0}+w_{1} x_{1}+\cdots+w_{k} x_{k}$, we pick $i>0$ such that $\left|w_{i}\right|$ is the largest among $\left|w_{1}\right|, \ldots,\left|w_{k}\right|$, and normalize it to the

\footnotetext{
7 According to the experimental results reported later, however, the three-layer NN as depicted in Figure 1 also seems to be a good alternative. It is left for future work to test whether NNs with more than four layers are useful for our task.

${ }^{8}$ We actually used $-\log \left(\max \left(1-\left|o_{i}-\ell_{i}\right|, \epsilon\right)\right)$ for a small positive number $\epsilon$, to avoid the overflow of floating point arithmetic.

${ }^{9}$ We plan to replace this with a more standard one based on continued fraction expansion.
} 
form $w_{0}^{\prime}+w_{1}^{\prime} x_{1}+\cdots+w_{k}^{\prime} x_{k}$ where $w_{j}^{\prime}=w_{j} / w_{i}$ (thus, $0 \leq\left|w_{j}^{\prime}\right| \leq 1$ for $j>0$ ). We then pick $-K<n_{j}, m_{j}<K$ such that $n_{j} / m_{j}$ is closest to $\left|w_{j}^{\prime}\right|$, and obtain $w_{0}^{\prime}+\left(n_{1} / m_{1}\right) x_{1}+\cdots+\left(n_{k} / m_{k}\right) x_{k}$. Finally, by multiplying the expression with the least common multiple $M$ of $m_{1}, \ldots, m_{k}$, and rounding $M w_{0}^{\prime}$ with an integer $c_{0}$, we obtain $c_{0}+M\left(n_{1} / m_{1}\right) x_{1}+\cdots+M\left(n_{k} / m_{k}\right) x_{k}$.

If too many qualifiers are extracted (typically when the number of hidden nodes is large; note that qualifiers are extracted from each hidden node), we prioritize them by inspecting the weights of the third and fourth layers, and possibly discard those with low priorities. The priority of qualifiers obtained from the $i$-th hidden node $N_{2, i}$ in the second layer is set to $p_{i}=\sum_{j}\left|w_{3, i, j} w_{4, j, 1}\right|$, The priority $p_{i}$ estimates the influence of the value of the $i$-th hidden node, hence the importance of the corresponding qualifiers.

Formula Synthesis. This phase no longer uses the trained NN and simply applies a classical method (e.g. the Quine-McCluskey method, if the number of candidate qualifiers is small) for synthesizing a Boolean formula from a truth table (with don't care inputs). ${ }^{10}$ Given qualifiers $Q_{1}, \ldots, Q_{m}$ and $\mathrm{P} / \mathrm{N}$ examples $d_{1}, \ldots, d_{n}$ with their labels $\ell_{1}, \ldots, \ell_{n}$, the goal is to construct a Boolean combination $\varphi$ of $Q_{1}, \ldots, Q_{m}$ such that $\varphi\left(d_{i}\right)=\ell_{i}$ for every $i$. To this end, we just need to construct a truth table where each row consists of $Q_{1}\left(d_{i}\right), \ldots, Q_{m}\left(d_{i}\right), \ell_{i}(i=1, \ldots, n)$, and obtain a Boolean function $f\left(b_{1}, \ldots, b_{m}\right)$ such that $f\left(Q_{1}\left(d_{i}\right), \ldots, Q_{m}\left(d_{i}\right)\right)=\ell_{i}$ for every $i$, and let $\varphi$ be $f\left(Q_{1}, \ldots, Q_{m}\right)$. Table 2 gives an example, where $m=2$, $Q_{1}=x+2 y>0$ and $Q_{2}=x-y<0$. In this case, $f\left(b_{1}, b_{2}\right)=b_{1} \wedge b_{2}$, hence $\varphi=x+2 y>0 \wedge x-y<0$.

One may think that we can also use information on the weights of the third and fourth layers of the trained NN in the formula synthesis phase. Our rationale for not doing so is as follows.

- Classically (i.e. without using NNs) synthesizing a Boolean function is not so costly; it is relatively much cheaper than the task of finding relevant qualifiers in the previous phase.

- It is not so easy to determine from the weights what Boolean formula is represented by each node of a NN. For example, as discussed earlier, $x \wedge y$ and $x \vee y$ can be represented by $\sigma\left(\beta\left(x+y-\frac{3}{2}\right)\right)$ and $\sigma\left(\beta\left(x+y-\frac{1}{2}\right)\right)$, whose difference is subtle (only the additive constants differ).

- By only using partial information about the trained NN, we need not worry too much about the problem of overfitting. Indeed, as discussed later in Section 2.2 and Appendix A.3, a form of overfitting is observed also in our experiments, but even in such cases, we could extract useful qualifiers.

- As confirmed by the experiments reported later, not all necessary qualifiers may be extracted in the previous phase; in such a case, trying to extract a Boolean formula directly from the NN would fail. By using a classical approach to a Boolean function synthesis, we can easily take into account the qualifiers

$\overline{10}$ The current implementation uses an ad hoc, greedy method, which will be replaced by a more standard one for Boolean decision tree construction. 
Table 2. An Example of Truth Tables Constructed from Qualifiers and Examples

\begin{tabular}{|l|l|l|l|}
\hline$d_{i}$ & $x+2 y>0$ & $x-y<0$ & $\ell_{i}$ \\
\hline$(1,0)$ & 1 & 1 & 1 \\
$(1,1)$ & 1 & 0 & 0 \\
$\cdots$ & $\ldots$ & $\cdots$ & $\cdots$ \\
$(-2,-1)$ & 0 & 1 & 0 \\
$(-2,-2)$ & 0 & 0 & 0 \\
\hline
\end{tabular}

collected by other means (e.g., in the context of program invariant synthesis, we often collect relevant qualifiers from conditional expressions in a given program).

Nevertheless, it is reasonable to use the weights of the second and third layers of the trained NN as hints for the synthesis of $\varphi$, which is left for future work.

\subsection{Experiments}

We have implemented a tool called NeuGuS based on the method described above using ocaml-torch ${ }^{11}$, OCaml interface for the PyTorch library, and conducted experiments. The source code of our tool is available at https://github. $\mathrm{com} /$ naokikob/neugus. All the experiments were conducted on a laptop computer with Intel(R) Core(TM) i7-8650U CPU (1.90GHz) and 16 GB memory; GPU was not utilized for training NNs.

Learning Conjunctive Formulas. As a warming-up, we have randomly generated a conjunctive formula $\varphi(x, y)$ of the form $A \wedge B$ where $A$ and $B$ are linear inequalities of the form $a x+b y+c>0$, and $a, b$ and $c$ are integers such that $-4 \leq a, b \leq 4$ and $-9 \leq c \leq 9$ with $a b \neq 0$. We set $P=\{(x, y) \in$ $\left.\mathbf{Z}^{2} \cap([-25,25] \times[-25,25]) \| \varphi(x, y)\right\}$ and $N=\left\{(x, y) \in \mathbf{Z}^{2} \cap([-25,25] \times\right.$ $[-25,25]) \| \neg \varphi(x, y)\}$ as the sets of positive and negative examples, respectively (thus, $|P \cup N|=51 \times 51=2601$ ). ${ }^{12}$ The left-hand side of Figure 4 plots positive examples for $A \equiv-2 x-y+4>0$ and $B \equiv 3 x-4 y+5>0$. We have randomly generated 20 such formula instances, and ran our tool three times for each instance to check whether the tool could find qualifiers $A$ and $B$. In each run, the NN training was repeated either until the accuracy becomes $100 \%$ and the loss becomes small enough, or until the number of training steps (the number of forward and backward propagations) reaches 30,000. If the accuracy does not reach $100 \%$ within 30,000 steps or the loss does not become small enough (less than $10^{-4}$ ), the tool retries the NN training from scratch, up to three times. (Even if the accuracy does not reach $100 \%$ after three retries, the tool proceeds to the next phase for qualifier discovery.) As the optimizer, we have

11 https://github.com/LaurentMazare/ocaml-torch.

${ }^{12}$ We have excluded out instances where $A$ or $B$ is subsumed by the other, and those where the set of positive or negative examples is too small. 

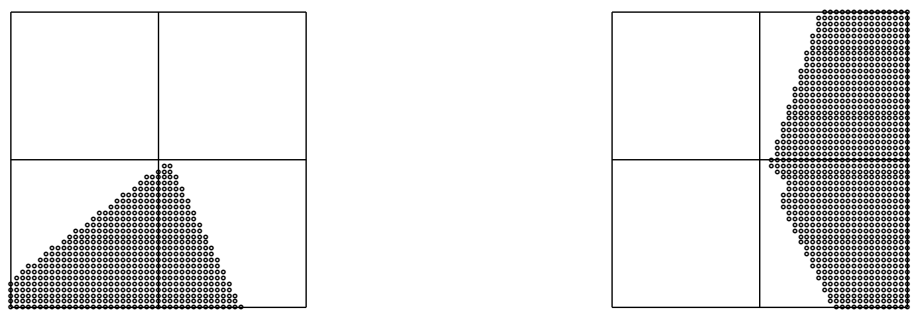

Fig. 4. Visualization of sample instances: $-2 x-y+4>0 \wedge 3 x-4 y+5>0$ (left) and $(2 x+y+1>0 \wedge x-y-9>0) \vee(x+y>0 \wedge 3 x-y-5>0)$ (right, Instance \#10 for $(A \wedge B) \vee(C \wedge D))$. The small circles show positive examples, and the white area is filed with negative examples.

used Adam [12] with the default setting of ocaml-torch $\left(\beta_{1}=0.9, \beta_{2}=0.999\right.$, no weight decay), and the learning rate was 0.001 . We did not use mini-batch training; all the training data were given at each training step.

Table 3 shows the result of experiments. The meaning of each column is as follows:

- "\#hidden nodes": the number of hidden nodes. " $m_{1}: m_{2}$ " means that a fourlayer NN was used, and that the numbers of hidden nodes in the second and third layers are respectively $m_{1}$ and $m_{2}$, while " $m$ " means that a three-layer (instead of four-layer) NN was used, with the number of hidden nodes being $m$.

- "loss func.": the loss function used for training. "log" means $\frac{1}{n} \sum_{i=1}^{n}-\log (1-$ $\left.\left|o_{i}-\ell_{i}\right|\right)$ (where $o_{i}$ and $\ell_{i}$ are the prediction and label for the $i$-th example), and "mse" means the mean square error function.

- "\#retry": the total number of retries. For each problem instance, up to 3 retries were performed. (Thus, there can be 20 (instances) $\times 3$ (runs) $\times$ 3 (retries per run) $=180$ retires in total at maximum.)

- "\%success": the percentage of runs in which a logical formula that separates positive and negative examples was constructed. The formula may not be identical to the original formula used to generate the $\mathrm{P} / \mathrm{N}$ examples (though, for this experiment of synthesizing $A \wedge B$, all the formulas synthesized were identical to the original formulas).

- "\%qualifiers": the percentage of the original qualifiers (i.e., inequalities $A$ and $B$ in this experiment) found.

- "\#candidates": the average number of qualifier candidates extracted from the NN. Recall that from each hidden node in the first layer, we extract three inequalities $\left(c_{0}+c_{1} x_{1}+\cdots+c_{k} x_{k}>e\right.$ for $\left.e \in\{0,-1,1\}\right)$; thus, the maximum number of generated candidates is $3 m$ for a NN with $m$ hidden nodes in the second layer.

- "time": the average execution time per run. Note that the current implementation is naive and does not use GPU. 
Table 3. Experimental Results for Learning a Conjunctive Formula $A \wedge B$.

\begin{tabular}{|c|c|c|c|c|c|c|}
\hline \#hidden nodes & loss func. & \#retry & \%success & \%qualifiers & \#candidates & time (sec.) \\
\hline \hline $4: 4$ & $\log$ & 0 & $100 \%$ & $100 \%$ & 6.8 & 27.7 \\
\hline 4 & $\log$ & 0 & $100 \%$ & $100 \%$ & 6.7 & 25.2 \\
\hline
\end{tabular}

As shown in Table 3, our tool worked quite well; it could always find the original qualifiers, no matter whether the number of layers is three or four. ${ }^{13}$

Learning Formulas with Conjunction and Disjunction We have also tested our tool for formulas with both conjunction and disjunction. We have randomly generated 20 formulas of the form $(A \wedge B) \vee(C \wedge D),{ }^{14}$ where $A, B, C$, and $D$ are linear inequalities, and prepared the sets of positive and negative examples as in the previous experiment. The right-hand side of Figure 4 plots positive examples for $(2 x+y+1>0 \wedge x-y-9>0) \vee(x+y>0 \wedge 3 x-y-5>0)$. The visualization of all the 20 instances is given in Appendix A.1. As before, we ran our tool three times for each instance, with several variations of NNs.

The result of the experiment is summarized in Table 4, where the meaning of each column is the same as that in the previous experiment. Among four-layer NNs, 32:32 with the log loss function showed the best performance in terms of the columns \%success and \%qualifiers. However, 8:8 with the log function also performed very well, and is preferable in terms of the number of qualifier candidates generated (\#candidates). As for the two loss functions, the log function generally performed better; therefore, we use the log function in the rest of the experiments. The running time does not vary among the variations of NNs; the number of retries was the main factor to determine the running time.

As for three-layer NNs, the NN with 8 hidden nodes performed quite badly. This matches the intuition that the third layer alone is not sufficient for recognizing the nested conjunctions and disjunctions. To our surprise, however, the NN with 32 hidden nodes actually showed the best performance in terms of \%success and \%qualifiers, although \#candidates (smaller is better) is much larger than those for four-layer NNs. We have inspected the reason for this, and found that the three-layer $\mathrm{NN}$ is not recognizing $(A \wedge B) \vee(C \wedge D)$, but classify positive and negative examples by doing a kind of patchwork, using a large number of (seemingly irrelevant) qualifiers which happened to include the correct qualifiers $A, B, C$, and $D$; for interested readers, we report our analysis in Appendix A.3. We believe, however, four-layer NNs are preferable in general, due to the smaller

${ }^{13}$ We have actually tested our tool also with a larger number of nodes, but we omit those results since they were the same as the case for 4:4 and 4 shown in the table: $100 \%$ success rate and $100 \%$ qualifiers found.

14 After the generation, we have manually excluded instances that have simpler equivalent formulas (e.g. $(x+y>1 \wedge x+y>0) \vee(x-y>1 \wedge x-y>0)$ is equivalent to $x+y>1 \vee x-y>1$, hence removed), and regenerated formulas. 
Table 4. Experimental Results for Learning Formulas of the form $(A \wedge B) \vee(C \wedge D)$.

\begin{tabular}{|c|c|c|c|c|c|c|}
\hline \#hidden nodes & loss func. & \#retry & \%success & \%qualifiers & \#candidates & time (sec.) \\
\hline \hline $8: 8$ & $\log$ & 12 & $93.3 \%$ & $96.7 \%$ & 18.6 & 42.1 \\
\hline $8: 8$ & mse & 17 & $86.7 \%$ & $94.6 \%$ & 18.1 & 40.6 \\
\hline $16: 16$ & $\log$ & 0 & $91.7 \%$ & $95.8 \%$ & 26.2 & 32.9 \\
\hline $16: 16$ & $\operatorname{mse}$ & 3 & $85.0 \%$ & $92.5 \%$ & 26.9 & 31.7 \\
\hline $32: 32$ & $\log$ & 0 & $100 \%$ & $97.9 \%$ & 38.9 & 31.1 \\
\hline $32: 32$ & $\operatorname{mse}$ & 15 & $85.0 \%$ & $91.7 \%$ & 40.4 & 39.9 \\
\hline 8 & $\log$ & 156 & $36.7 \%$ & $66.3 \%$ & 21.6 & 92.4 \\
\hline 16 & $\log$ & 54 & $95.0 \%$ & $96.7 \%$ & 37.1 & 55.8 \\
\hline 32 & $\log$ & 2 & $100 \%$ & $98.3 \%$ & 58.5 & 36.3 \\
\hline
\end{tabular}

numbers of qualifier candidates generated. Three-layer NNs can still be a choice, if program or invariant synthesis tools (that use our tool as a backend for finding qualifiers) work well with a very large number of candidate qualifiers; for example, the ICE-learning-based CHC solver HoICE [4] seems to work well with 100 candidate qualifiers (but probably not for 1000 candidates).

As for the 32:32 four-layer NN (which performed best among four-layer NNs), only 5 correct qualifiers were missed in total and all of them came from Instance \#10 shown on the right-hand side of Figure 4. Among the four qualifiers, $x-$ $y-9>0$ was missed in all the three runs, and $x+y>0$ was missed in two of the three runs; this is somewhat expected, as it is quite subtle to recognize the line $x-y-9=0$ also for a human being. The $\mathrm{NN}$ instead found the qualifiers like $x>6$ and $y<-5$ to separate the positive and negative examples. We were rather surprised that the $\mathrm{NN}$ could recognize the correct qualifiers for other subtle instances like \#2 and \#13 in Appendix A.1.

Figure 5 shows the effect of the prioritization of qualifiers, as discussed in Section 2.1. We have sorted the hidden nodes in the second layer based on the weights of the third and fourth layers, and visualized, in the graph on the lefthand side, the average number of correct qualifiers (per run) extracted from top $50 \%, 75 \%$, and $100 \%$ of the hidden nodes. The graph on the right-hand side shows the average number of candidate qualifiers (per run) extracted from top $50 \%, 75 \%$, and $100 \%$ of the hidden nodes. As can be seen in the graphs, while the number of candidate qualifiers is almost linear in the number of hidden nodes considered, most of the correct qualifiers were found from top $75 \%$ of the hidden nodes. This justifies our strategy to prioritize qualifier candidates based on the weights of the third and fourth layers.

Appendix A.4 also reports experimental results to compare the sigmoid function with other activation functions.

To check whether our method scales to larger formulas, we have also tested our tool for formulas of the form $(A \wedge B \wedge C) \vee(D \wedge E \wedge F) \vee(G \wedge H \wedge I)$. where $A, \ldots, I$ are linear inequalities of the form $a x+b y+c>0$, and $a$ and 

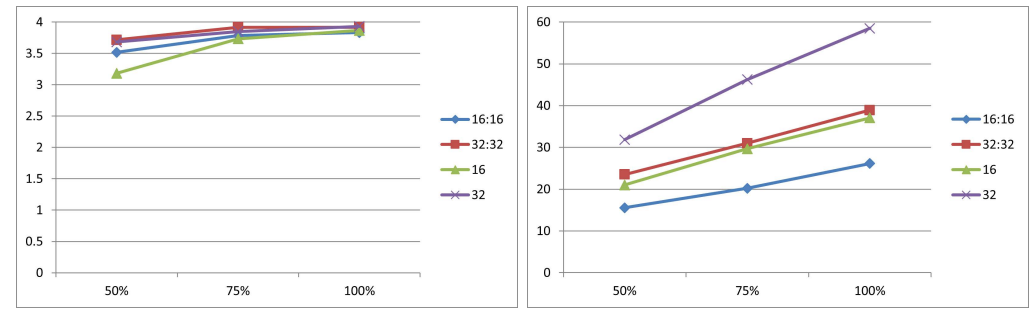

Fig. 5. The number of correct qualifiers discovered (left) and the number of qualifier candidates generated (right). Most of the correct qualifiers appear in top $50 \%$ of the candidates.

Table 5. Experimental Results for Learning Formulas of the form $(A \wedge B \wedge C) \vee(D \wedge$ $E \wedge F) \vee(G \wedge H \wedge I)$.

\begin{tabular}{|c|c|c|c|c|c|}
\hline \#hidden nodes & \#retry & \%success & \%qualifiers & \#candidates & time (sec.) \\
\hline \hline 32 & 37 & $83.3 \%$ & $86.5 \%$ & 126.6 & 78.7 \\
\hline $32: 4$ & 13 & $78.3 \%$ & $84.6 \%$ & 127.3 & 79.6 \\
\hline $32: 32$ & 0 & $70.0 \%$ & $79.6 \%$ & 134.1 & 71.1 \\
\hline 64 & 1 & $100 \%$ & $90.2 \%$ & 126.6 & 200.1 \\
\hline $64: 16$ & 0 & $96.7 \%$ & $86.7 \%$ & 204.9 & 86.1 \\
\hline $64: 64$ & 0 & $91.7 \%$ & $84.1 \%$ & 215.3 & 107.6 \\
\hline
\end{tabular}

$b$ are integers such that $-4 \leq a, b \leq 4$ and $-30 \leq c \leq 30$ with $a b \neq 0$. We set $P=\left\{(x, y) \in \mathbf{Z}^{2} \cap([-30,30] \times[-30,30]) \|=\varphi(x, y)\right\}$ and $N=\{(x, y) \in$ $\left.\mathbf{Z}^{2} \cap([-30,30] \times[-30,30]) \| \neg \varphi(x, y)\right\}$ as the sets of positive and negative examples, respectively. We have collected 20 problem instances, by randomly generating formulas and then manually filtering out those that have a simpler representation than $(A \wedge B \wedge C) \vee(D \wedge E \wedge F) \vee(G \wedge H \wedge I)$. Table 5 summarizes the experimental results. We have used the log function as the loss function. For the qualifier extraction, we extracted five (as opposed to three, in the experiments above) inequalities of the form $\left(c_{0}+c_{1} x_{1}+\cdots+c_{k} x_{k}>e\right.$ for $\left.e \in\{-2,-1,0,1,2\}\right)$ from each hidden node. The threshold for the loss was set to $10^{-5}$, except that it was set to $10^{-4}$ for the three-layer NN with 32 nodes.

The result indicates that our method works reasonably well even for this case, if hyper-parameters (especially, the number of nodes) are chosen appropriately; how to adjust the hyper-parameters is left for future work. Interestingly, the result tends to be better for NNs with a smaller number of nodes in the third layer. Our rationale for this is that a smaller number of nodes in the third layer forces the nodes in the second layer more strongly to recognize appropriate features. 


\subsection{Extensions for Non-Linear Constraints}

We can easily extend our approach to synthesize formulas consisting of non-linear constraints such as polynomial constraints of a bounded degree and modulo operations. For that purpose, we just need to add auxiliary inputs like $x^{2}, x \bmod 2$ to a NN. We have tested our tool for quadratic inequalities of the form $a x^{2}+b x y+$ $c y^{2}+d x+e y+f>0$ (where $-4 \leq a, b, c, d, e \leq 4$ and $-9 \leq f \leq 9$; for ovals, we allowed $f$ to range over $[-200,199]$ because there are only few positive examples for small values of $f$ ), and their disjunctions. For a quadratic formula $\varphi(x, y)$, we set $P=\left\{\left(x, y, x^{2}, x y, y^{2}\right) \mid(x, y) \in \mathbf{Z}^{2} \cap([-20,20] \times[-20,20]), \models \varphi(x, y)\right\}$ and $N=\left\{\left(x, y, x^{2}, x y, y^{2}\right) \mid(x, y) \in \mathbf{Z}^{2} \cap([-20,20] \times[-20,20]), \models \neg \varphi(x, y)\right\}$ as the sets of positive and negative examples, respectively.

The table below shows the result for a single quadratic inequality. We have prepared four instances for each of ovals, parabolas, and hyperbolas; the instances used in the experiment are visualized in Appendix A.2 (Instances \#1\#12). As can be seen in the table, the tool worked very well.

\begin{tabular}{|c|c|c|c|c|c|c|}
\hline \#hidden nodes & loss func. & \#retry & \% success & \% qualifiers & \#candidates & time (sec.) \\
\hline 4 & $\log$ & 0 & $100 \%$ & $100 \%$ & 7.4 & 22.2 \\
\hline 8 & $\log$ & 0 & $100 \%$ & $100 \%$ & 10.7 & 18.3 \\
\hline
\end{tabular}

We have also prepared six instances of formulas of the form $A \vee B$, where $A$ and $B$ are quadratic inequalities; they are visualized as Instances \#13-\#18 in Appendix A.2. The result is shown in the table below. The NN with 32 nodes performed reasonably well, considering the difficulty of the task. All the failures actually came from Instances \#16 and \#18, and the tool succeeded for the other instances. For \#16, the tool struggled to correctly recognize the oval $-2 x^{2}-2 x y-$ $2 y^{2}-3 x-4 y+199>0\left(-2 x^{2}-2 x y-2 y^{2}-3 x-4 y+197>0\right.$ was instead generated as a candidate; they differ at only two points $(0,9)$ and $(0,-11))$, and for $\# 18$, it failed to recognize the hyperbola $-4 x^{2}-4 x y+y^{2}+2 x-2 y-2>0$.

\begin{tabular}{|c|c|c|c|c|c|c|}
\hline \#hidden nodes & loss func. & \#retry & \%success & \%qualifiers & \#candidates & time (sec.) \\
\hline 8 & $\log$ & 5 & $50 \%$ & $52.8 \%$ & 22.1 & 43.9 \\
\hline 16 & $\log$ & 0 & $44.4 \%$ & $55.6 \%$ & 38.6 & 26.1 \\
\hline 32 & $\log$ & 0 & $66.7 \%$ & $80.6 \%$ & 72.1 & 25.4 \\
\hline
\end{tabular}

\section{Predicate Synthesis from Implication Constraints and its Application to CHC Solving}

We have so far considered the synthesis of logical formulas in the classical setting of supervised learning of classification, where positive and negative examples are given. In the context of program verification, we are also given so called implication constraints [6,4], like $p(1) \Rightarrow p(2)$, which means "if $p(1)$ is a positive example, so is $p(2)$ " (but we do not know whether $p(1)$ is a positive or negative example). As advocated by Garg et al. [6], implication constraints play an important role in the discovery of an inductive invariant (i.e. an invariant 
Inv that satisfies a certain condition of the form $\forall x, y \cdot(\psi(\operatorname{Inv}, x, y) \Rightarrow \operatorname{Inv}(x))$; for a state transition system, $\psi(\operatorname{Inv}, x, y)$ is of the form $\operatorname{Inv}(y) \wedge R(y, x)$ where $R$ is the transition relation). As discussed below, our framework of NN-guided synthesis can easily be adapted to deal with implication constraints. Implication constraints are also called implication examples below.

\subsection{The Extended Synthesis Problem and Our Method}

We first define the extended synthesis problem. A predicate signature is a map from a finite set $P \operatorname{Var}=\left\{p_{1}, \ldots, p_{m}\right\}$ of predicate variables to the set Nat of natural numbers. For a predicate signature $\operatorname{Ar}, \operatorname{Ar}\left(p_{i}\right)$ denotes the arity of the predicate $p_{i}$. We call a tuple of the form $\left(p_{i}, n_{1}, \ldots, n_{\operatorname{Ar}\left(p_{i}\right)}\right)$ with $n_{j} \in \mathbf{Z}$ an atom, and often write $p_{i}\left(n_{1}, \ldots, n_{\operatorname{Ar}\left(p_{i}\right)}\right)$ for it; we also write $\widetilde{n}$ for a sequence $n_{1}, \ldots, n_{\operatorname{Ar}\left(p_{i}\right)}$ and write $p_{i}(\widetilde{n})$ for the atom $p_{i}\left(n_{1}, \ldots, n_{\operatorname{Ar}\left(p_{i}\right)}\right)$. We write $\mathbf{A t o m s}_{\operatorname{Ar}}$ for the set of atoms consisting of the predicates given by the signature Ar.

Definition 2 (Predicate synthesis problem with Implication Examples). The goal of the predicate synthesis problem with positive/negative/implication examples (the PNI synthesis problem, for short) is, given:

1. a signature Ar $\in P$ Var $\rightarrow$ Nat; and

2. a set $I$ of implication examples of the form $a_{1} \wedge \cdots \wedge a_{k} \Rightarrow b_{1} \vee \cdots \vee b_{\ell}$ where $a_{1}, \ldots, a_{k}, b_{1}, \ldots, b_{\ell} \in \mathbf{A t o m s}_{\mathrm{Ar}}$, and $k+\ell>0$ (but $k$ or $\ell$ may be 0 )

as an input, to find a map $\theta$ that assigns, to each $p_{i} \in P$ Var, a logical formula $\varphi_{i}\left(x_{1}, \ldots, x_{\operatorname{Ar}\left(p_{i}\right)}\right)$ such that $\models \theta a_{1} \wedge \cdots \wedge \theta a_{k} \Rightarrow \theta b_{1} \vee \cdots \vee \theta b_{\ell}$ holds for each implication example $a_{1} \wedge \cdots \wedge a_{k} \Rightarrow b_{1} \vee \cdots \vee b_{\ell} \in I$. Here, for an atom $a=$ $p\left(n_{1}, \ldots, n_{j}\right), \theta a$ is defined as $(\theta p)\left[n_{1} / x_{1}, \ldots, n_{j} / x_{j}\right]$. We call an implication example of the form $\Rightarrow b$ ( $a \Rightarrow$, resp.) as a positive (negative, resp.) example, and write $P \subseteq I$ and $N \subseteq I$ for the sets of positive and negative examples respectively.

Example 1. Let $\operatorname{Ar}=\{p \mapsto 1, q \mapsto 1\}$ and $I$ be $P \cup N \cup I^{\prime}$ where:

$$
\begin{aligned}
P= & \{\Rightarrow p(0), \Rightarrow q(1)\} \quad N=\{p(1) \Rightarrow, q(0) \Rightarrow\} \\
I^{\prime}= & \{p(2) \Rightarrow q(3), q(3) \Rightarrow p(4), p(2) \wedge q(2) \Rightarrow, p(3) \wedge q(3) \Rightarrow, \\
& p(4) \wedge q(4) \Rightarrow, \Rightarrow p(2) \vee q(2), \Rightarrow p(3) \vee q(3), \Rightarrow p(4) \vee q(4)\}
\end{aligned}
$$

Then $\theta=\left\{p \mapsto x_{1} \bmod 2=0, q \mapsto x_{1} \bmod 2=1\right\}$ is a valid solution for the synthesis problem $(\mathrm{Ar}, I)$.

We generalize the method described in Section 2.1 as follows.

1. Prepare a $\mathrm{NN} \mathcal{N}_{i}$ for each predicate variable $p_{i} . \mathcal{N}_{i}$ has $\operatorname{Ar}\left(p_{i}\right)$ input nodes (plus additional inputs, if we aim to generate non-linear formulas, as discussed in Section 2.3). For an atom $a \equiv p_{i}(\widetilde{n})$, we write $o_{a}$ for the output of $\mathcal{N}_{i}$ for $\widetilde{n}$ below (note that the value of $o_{a}$ changes during the course of training). 
2. Train all the NNs $\mathcal{N}_{1}, \ldots, \mathcal{N}_{m}$ together. For each atom $p_{i}(\widetilde{n})$ occurring in implication examples, $\widetilde{n}$ is used as a training datum for $\mathcal{N}_{i}$. For each implication example $e \equiv a_{1} \wedge \cdots \wedge a_{k} \Rightarrow b_{1} \vee \cdots \vee b_{\ell} \in I$, we define the loss $\operatorname{loss}_{e}$ for the example by:

$$
\operatorname{loss}_{e}=-\log \left(1-\prod_{i=1}^{k} o_{a_{i}} \cdot \prod_{j=1}^{\ell}\left(1-o_{b_{j}}\right)\right) .{ }^{15}
$$

The idea is to ensure that the value of $l_{0 s s_{e}}$ is 0 if one of $o_{a_{i}}$ 's is 0 or one of $o_{b_{j}}$ 's is 1 , and that the value of $\operatorname{loss}_{e}$ is positive otherwise. This reflects the fact that $a_{1} \wedge \cdots \wedge a_{k} \Rightarrow b_{1} \vee \cdots \vee b_{\ell}$ holds just if one of $a_{i}$ 's is false or one of $b_{j}$ 's is true. Note that the case where $k=0$ and $\ell=1(k=1$ and $\ell=0$, resp.) coincides with the logarithmic loss function for positive (negative, resp.) examples in Section 2.1. Set the overall loss of the current NNs as the average of loss $_{e}$ among all the implication constraints, and use the gradient descent to update the weights and biases of NNs. Repeat the training until all the implication constraints are satisfied (by considering values greater than 0.5 as true, and those less than 0.5 as false).

3. Extract a set $Q_{i}$ of qualifiers from each trained $\mathcal{N}_{i}$, as in Section 2.1.

4. Synthesize a formula for the predicate $p_{i}$ as a Boolean combination of $Q_{i}$. This phase is also the same as Section 2.1, except that, as the label $\ell_{a}$ for each atom $a$, we use the prediction of the trained NNs. Note that unlike in the setting of the previous section where we had only positive and negative examples, we may not know the correct label of some atom due to the existence of implication examples. For example, given $p(0) \vee p(1) \Rightarrow$ and $\Rightarrow p(0) \vee p(1)$, we do not know which of $p(0)$ and $p(1)$ should hold. We trust the output of the trained NN in such a case. Since we have trained the NNs until all the implication constraints are satisfied, it is guaranteed that, for positive and negative examples, the outputs of NNs match the correct labels. Thus, the overall method strictly subsumes the one described in Section 2.1.

\subsection{Preliminary Experiments}

We have extended the tool based on the method described above, and tested it for several examples. We report some of them below.

As a stress test for implication constraints, we have prepared the following input, with no positive/negative examples (where $\operatorname{Ar}=\{p \mapsto 1\}$ ):

$$
\begin{aligned}
& I=\{p(2 n) \wedge p(2 n+1) \Rightarrow \mid n \in[-10,10]\} \\
& \cup\{\Rightarrow p(2 n) \vee p(2 n+1) \mid n \in[-10,10]\}
\end{aligned}
$$

The implication examples mean that, for each integer $n \in[-10,10]$, exactly one of $p(2 n)$ and $p(2 n+1)$ is true. We ran our tool with an option to enable the

${ }^{15}$ In the implementation, we approximated $l_{\text {oss }}$ by $-\log \max \left(\epsilon, 1-\prod_{i=1}^{k} o_{a_{i}} \cdot \prod_{j=1}^{\ell}(1-\right.$ $\left.o_{b_{j}}\right)$ ) for a small positive number $\epsilon$ in order to avoid an overflow of the floating point arithmetic. 


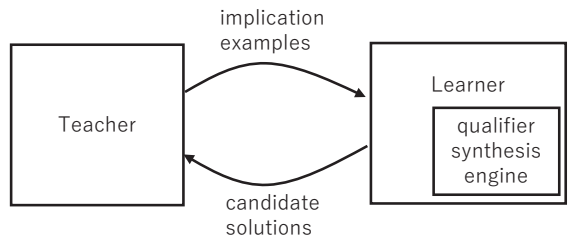

Fig. 6. Inside HoICE

"mod 2" operation, and obtained $\left\{p \mapsto x_{1} \bmod 2<1\right\}$ as a solution (which is correct; another solution is $x_{1} \bmod 2>0$ ).

We have also tested our tool for several instances of the CHC (Constrained Horn Clauses) solving problem [3]. A constrained Horn clause is a formula of the form $A_{1} \wedge \cdots \wedge A_{k} \Rightarrow A_{0}$, where each $A_{i}$ is a constraint (of linear arithmetic in this paper) or an atomic formula of the form $p\left(e_{1}, \ldots, e_{k}\right)$ where $p$ is a predicate variable. The goal of $\mathrm{CHC}$ solving is, given a set of constrained Horn clauses, to check whether there is a substitution for predicate variables that makes all the clauses valid (and if so, output a substitution). Various program verification problems [3], as well as the problem of finding inductive invariants, can be reduced to the problem of $\mathrm{CHC}$ solving. Various CHC solvers $[14,9,24,4]$ have been implemented so far, and HoIce [4] is one of the state-of-the-art solvers, which is based on the ICE-learning framework. As illustrated in Figure 6, HoICE consists of two main components: a teacher and a learner. The learner generates a candidate solution (a map from predicate variables to formulas) from implication examples, and the teacher checks whether the candidate solution is valid (i.e. satisfies all the clauses) by calling SMT solvers, and if not, generates a new implication example. The learner has a qualifier synthesis engine and combines it with a method for Boolean decision tree construction [7]. The main bottleneck of HoICE has been the qualifier synthesis engine, and the aim of our preliminary experiments reported below is thus to check whether our NN-guided synthesis method can be used to reduce the bottleneck.

To evaluate the usefulness of our NN-guided synthesis, we have implemented the following procedure, using HoICE and our NN-guided synthesis tool (called NeuGuS) as backends.

Step 1: Run HoIce for 10 seconds to collect implication examples.

Step 2: Run NeuGuS to learn qualifiers.

Step 3: Re-run HoIcE with the qualifiers as hints (where the time limit is initially set to 10 seconds).

Step 4: If Step 3 fails, collect implication examples from the execution of Step 3, and go back to Step 2, with the time limit for HoIcE increased by 5 seconds.

In Step 2, we used a four-layer NN, and set the numbers of hidden nodes in the second and third layers to 4 . When NEuGuS returns a formula, the inequality constraints that constitute the formula as qualifiers are passed to Step 3; other- 
wise, all the qualifier candidates extracted from the trained NNs are passed to Step 3.

We have collected the following $\mathrm{CHC}$ problems that plain HoICE cannot solve.

- Two problems that arose during our prior analysis of the bottleneck HoICE (plus, plusminus). The problem plusminus consists of the following CHCs.

$$
\begin{aligned}
\operatorname{plus}(m, n, r) \wedge \operatorname{minus}(r, n, s) & \Rightarrow m=s & & \operatorname{true} \Rightarrow \operatorname{plus}(m, 0, m) \\
n>0 \wedge \operatorname{plus}(m, n-1, r) & \Rightarrow \operatorname{plus}(m, n, r+2) & & \operatorname{true} \Rightarrow \operatorname{minus}(m, 0, m) \\
n>0 \wedge \operatorname{minus}(m, n-1, r) & \Rightarrow \operatorname{minus}(m, n, r-2) & &
\end{aligned}
$$

The above CHC is satisfied for plus $(m, n, r) \equiv r=m+2 n, \operatorname{minus}(m, n, r) \equiv r=$ $m-2 n$. The ICE-based CHC solver HoICE [4], however, fails to solve the CHC (and runs forever), as HoICE is not very good at finding qualifiers involving more than two variables.

- The five problems from the Inv Track of SyGus 2018 Competition (https: //github.com/SyGuS-Org/benchmarks/tree/master/comp/2018/Inv_Track, cggmp2005_variant_true-unreach-call_true-termination, jmbl_cggmp-new, fib_17n, fib_32, and jmbl_hola.07, which are named cggmp, cggmp-new, fib17, fib32, and hola.07 respectively in Table 3.2); the other problems can be solved by plain HoIce, hence excluded out).

- The problem \#93 from the code2inv benchmark set used in [22] (93; the other 123 problems can be solved by plain HoICE within 60 seconds).

- Two problems (pldi082_unbounded1.ml and xyz.ml) from the benchmark set (https://github.com/nyu-acsys/drift) of Drift [19], ${ }^{16}$ with a variant xyz_v.ml of $\mathrm{xyz} \cdot \mathrm{ml}$, obtained by generalizing the initial values of some variables.

The implementation and the benchmark set described above are available at https://github.com/naokikob/neugus.

Table 3.2 summarizes the experimental results. The experiments were conducted on the same machine as those of Section 2.1. We used HoICE 1.8.3 as a backend. We used 4-layer NNs with 32 and 8 hidden nodes in the second and third layers respectively. The column '\#pred' shows the number of predicates in the CHC problem, and columns '\#P', '\#N', and '\#I' respectively show the numbers of positive, negative, and implication examples (the maximum numbers in the three runs). The column 'Cycle' shows the minimum and maximum numbers of Step 2 in the three runs. The column 'Time' shows the execution time in seconds (which is dominated by NeuGuS), where the time is the average for three runs, and '-' indicates a time-out (with the time limit of 600 seconds). The next column shows key qualifiers found by NEuGuS. For comparison with our combination of HoICE and NEUGuS, the last column shows the times (in seconds) spent by Z3 (as a CHC solver) for solving the problems. ${ }^{17}$

16 The tool r_type was used to extract CHCs. The source programs have been slightly modified to remove Boolean arguments from predicates.

${ }^{17}$ Recall that our benchmark set collects only the problems that plain HoIcE cannot solve: although many of those problems can be solved by Z3 [14] much more quickly, there are also problems that Z3 cannot solve but (plain) HoIcE can. 
Table 6. Experimental results on CHC solving

\begin{tabular}{|l|r|r|r|r|c|c|c|c|}
\hline Problem & \#pred & \#P & \#N & \#I & Cycle & Time & Key qualifiers found & Z3 \\
\hline plus & 1 & 29 & 48 & 35 & 1 & 23.8 & $x_{0}+2 x_{1}+x_{2}=0$ & - \\
\hline plusminus & 2 & 90 & 137 & 42 & 2 & 63.2 & $x_{0}+2 x_{1}-x_{1}=0$ & - \\
\hline cggmp & 1 & 25 & 95 & 11 & 1 & 20.0 & $x_{0}+2 x_{1}-x_{2}=0$ & 0.05 \\
\hline cggmp-new & 1 & 8 & 97 & 15 & 1 & 24.1 & $x_{0}+2 x_{1} \geq 40, x_{0}+2 x_{1} \leq 42$ & 0.12 \\
\hline fib17n & 1 & 44 & 139 & 35 & $7-10$ & 349.7 & $-x_{0}+x_{2}-x_{3} \geq 0$ & 0.14 \\
\hline fib32 & 1 & 42 & 222 & 17 & - & - & & - \\
\hline fib32 -mod2 & 1 & 42 & 222 & 17 & $1-3$ & 53.7 & $x_{3} \bmod 2=0$ & - \\
\hline hola.07 & 1 & 78 & 314 & 12 & $2-3$ & 72.5 & $x_{0}+x_{1}=3 x_{2}$ & 0.05 \\
\hline codeinv93 & 1 & 32 & 260 & 23 & $2-3$ & 73.2 & $-3 x_{0}+x_{2}+x_{3}=0$ & 0.05 \\
\hline pldi082 & 2 & 15 & 42 & 34 & 1 & 24.6 & $2 x_{0}-x_{1}-x_{2} \geq-2$ & - \\
\hline xyz & 2 & 56 & 6 & 142 & - & - & & - \\
\hline xyz -gen & 2 & 251 & 139 & 0 & $3-5$ & 185.9 & $x_{0}+2 x_{1} \geq 0$ & 0.33 \\
\hline xyz_v & 2 & 160 & 303 & 43 & $2-8$ & 217.3 & $x_{0}-x_{1}-2 x_{2}+2 x_{3} \geq 0$ & - \\
\hline
\end{tabular}

The results reported above indicate that our tool can indeed be used to improve the qualifier discovery engine of ICE-learning-based CHC solver HoICE, which has been the main bottleneck of HoICE. With the default option, NEUGuS timed out for $f i b 32$ and xyz. For the problem $f i b 32$, the "mod 2" constraint is required. The row "fib32 -mod2" shows the result obtained by running NEuGuS with the "mod 2" constraint enabled. For the problem xyz, we discovered that the main obstacle was on the side of HoICE: HoICE (in the first step) finds no negative constraint for one of the two predicates, and no positive constraint for the other predicate; thus, NEUGuS returns "true" for the former predicate and "false" for the latter predicate. We have therefore prepared an option to eagerly collect learning data by applying unit-propagation to CHCs; the row "xyz -gen" shows the result for this option; see Appendix B for more detailed analysis of the problem xyz. Larger experiments on the application to CHC solvers are left for future work.

\section{Another Application: Programming with Oracles}

As another potential application of our framework of NN-guided synthesis of logical formulas, we propose a framework of programming with oracles. The overall flow of the framework is shown in Figure 7.

A programmer provides a program containing "oracles", along with the specification of the entire program, and the goal of the overall synthesis is to replace the oracles with appropriate code. Here is an example of a program with oracles and a specification:

let $\operatorname{abs} \mathrm{n}=$ if $\operatorname{oracle}(\mathrm{n})$ then $\mathrm{n}$ else $-\mathrm{n}$

let main $\mathrm{n}=\operatorname{assert}(\operatorname{abs}(\mathrm{n})>=0)$ (* specification of abs $*$ ) 


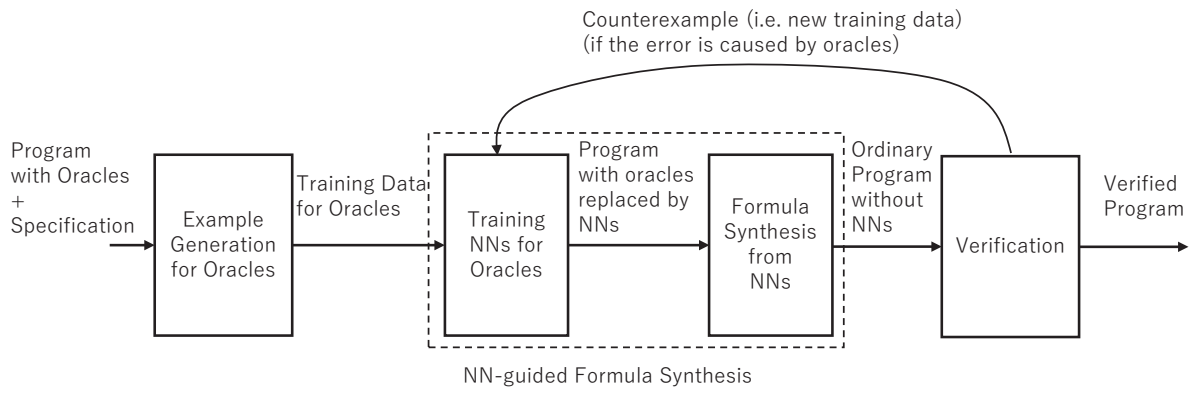

Fig. 7. Programming with Oracles

Here, the code for function abs contains an oracle, and the main function gives a specification of abs by using assertions (which says that for any integer $n$, $\operatorname{abs}(n) \geq 0$ should hold). The specification could alternatively be given in terms of example input/output pairs or pre/post conditions. (The specification could also be a soft one, like "minimize the running time".) The goal here is to replace $\operatorname{oracle}(\mathrm{n})$ with appropriate code, like $\mathrm{n}>0$. Thus, the framework can be viewed as a variation of the program synthesis from sketch [27], and the main new ingredient is the use of NNs to guide the synthesis. In the discussion below, we assume that oracles are restricted to those that return Boolean values. (To lift the restriction, we need to extended our formula synthesis method in Section 2 to synthesize arbitrary expressions.)

Each step in Figure 7 is described as follows.

1. Example generation: For each oracle in the program, we generate positive/negative/implication examples on oracles. This can be achieved by first replacing oracles with non-deterministic choice, and using random testing or model checking to obtain error traces (sequences of oracle calls) that violate the specification. For the abs program above, we can easily find that any of the following oracle calls leads to an assertion failure:

$$
\begin{aligned}
& \operatorname{oracle}(-1)=\text { true, } \operatorname{oracle}(-2)=\text { true }, \ldots \\
& \text { oracle }(1)=\text { false, } \operatorname{oracle}(2)=\text { false, } \ldots
\end{aligned}
$$

Thus, we can set $P=\{\Rightarrow \operatorname{oracle}(1), \Rightarrow \operatorname{oracle}(2), \ldots\}$ and

$N=\{\operatorname{oracle}(-1) \Rightarrow$, oracle $(-2) \Rightarrow, \ldots\}$ as positive and negative examples respectively.

In general, a single error trace may contain multiple calls of multiple oracles, like

$$
\operatorname{oracle}_{1}\left(d_{1}\right)=\text { true; oracle } e_{2}\left(d_{2}\right)=\text { false; } \text { oracle }_{1}\left(d_{3}\right)=\text { true }
$$

when a program contains multiple kinds of oracles and calls of them occur inside a recursion or loop. For the sequence of oracle calls above, we generate the following implication example:

$$
\neg \operatorname{oracle}_{1}\left(d_{1}\right) \vee \operatorname{oracle}_{2}\left(d_{2}\right) \vee \neg \operatorname{oracle}_{1}\left(d_{3}\right)
$$


(or, oracle $e_{1}\left(d_{1}\right) \wedge \operatorname{oracle}_{1}\left(d_{3}\right) \Rightarrow$ oracle $_{2}\left(d_{2}\right)$ in the syntax of implication examples in the previous section), which expresses that at least one of the return values of oracle calls is wrong.

One may also extract examples from successful traces. For example, given a successful trace

$$
\operatorname{oracle}_{1}\left(d_{4}\right)=\text { true; oracle } e_{2}\left(d_{5}\right)=\text { true, }
$$

we can add oracle $2\left(d_{5}\right) \Rightarrow \operatorname{oracle}_{1}\left(d_{4}\right)$ and oracle $\operatorname{or}_{1}\left(d_{4}\right) \Rightarrow \operatorname{oracle}_{2}\left(d_{5}\right)$ as implication examples. The reason why we have the conditions oracle $e_{2}\left(d_{5}\right) \Rightarrow \cdots$ and oracle $e_{1}\left(d_{4}\right) \Rightarrow \cdots$ is that it may be the case that both oracle $e_{1}\left(d_{3}\right)=$ true and oracle $e_{2}\left(d_{5}\right)=$ true were wrong but that the whole program execution succeeded just by coincidence. In general, from a successful trace:

$$
\operatorname{oracle}_{1}\left(d_{1}\right)=b_{1} ; \operatorname{oracle}_{2}\left(d_{2}\right)=b_{2} ; \cdots ; \operatorname{oracle}_{k}\left(d_{k}\right)=b_{k} \text {, }
$$

we add

$$
\bigwedge_{j \neq i}\left(\operatorname{oracle}_{j}\left(d_{j}\right)=b_{j}\right) \Rightarrow \operatorname{oracle}_{i}\left(d_{i}\right)=b_{i}
$$

for each $i \in\{1, \ldots, k\}$ as implication constraints. In general, however, we need to treat the examples generated from successful traces as soft constraints, to avoid a contradiction. For example, consider the following program:

let $f x=$ if $\operatorname{oracle}(x)$ then 0 else 0

let main $n=\operatorname{assert}(f \mathrm{n}=0$ )

In this case, the return value of oracle does not matter. Since both oracle $(0)=$ true and oracle $(0)=$ false are successful traces, we get $\Rightarrow$ oracle $(0)$ and oracle $(0) \Rightarrow$, which cannot be simultaneously satisfied; hence, they should be treated as soft constraints. The distinction between hard and soft constraints can be reflected in the NN training phase by adjusting the loss function (e.g. by giving small weights to the loss caused by soft constraints).

2. NN training: just as described in Section 3. Alternatively, one could directly verify NNs $[8,30,1,21,16]$ and use them as oracles, or "shield" NNs by ordinary code [32], without proceeding to the next phase for synthesis; these are also interesting directions, but outside the scope of the present paper.

3. Formula synthesis: this phase is also as described in Section 3.

4. Verification: Oracles are replaced with the formulas synthesized in the previous phase, and the resulting programs are verified and/or tested. If an error trace is found, generate implication constraints from it and go back to the training phase. If there is no oracle call sequence that avoids the error trace, then a bug is on the side of the program with oracles, hence report it to the programmer.

Our method for the NN-guided synthesis would serve as a key ingredient in the framework above. We have not yet implemented the framework, which is left for future work. To test the feasibility of the proposed framework, we have tested the procedure above for some benchmark programs of higher-order 
program verification ${ }^{18}$, by manually emulating the steps outside our NN-guided synthesis tool.

Among the most non-trivial examples was the synthesis of the condition of McCarthy's 91 function:

let $\operatorname{rec} \operatorname{mc91} \mathrm{x}=$ if $\operatorname{oracle}(\mathrm{x})$ then $\mathrm{x}-10$ else mc91 (mc91 $(\mathrm{x}+11))$

let main $\mathrm{n}=$ if $\mathrm{n}<=101$ then assert (mc91 $\mathrm{n}=91$ )

We could successfully synthesize the formula $x>100$ for oracle(x) above. More details are reported in Appendix C.

\section{Related Work}

There have recently been a number of studies on verification of neural networks $[8,30,1,21,16]$ : see [10] for an extensive survey. In contrast, the end goal of the present paper is to apply neural networks to verification and synthesis of classical programs. Closest to our use of NNs is the work of Ryan et al. [22,29] on Continuous Logic Network (CLN). CLN is a special neural network that imitates a logical formula (analogously like symbolic regressions discussed below), and it was applied to learn loop invariants. The main differences are: (i) The shape of a formula must be fixed in their original approach [22], while it need not in our method, thanks to our hybrid approach of extracting just qualifiers and using a classical method for constructing its Boolean combinations. Although their later approach [29] relaxes the shape restriction, it still seems less flexible than ours (in fact, the shape of invariants found by their tools seem limited, according to the code available at https://github.com/gryan11/cln2inv and https://github.com/jyao15/G-CLN). (ii) We consider a more general learning problem, using not only positive examples, but also negative and implication examples. This is important for applications to CHC solving discussed in Section 3 and oracle-based program synthesis in Appendix 4.

Finding inductive invariants has been the main bottleneck of program verification, both for automated tools (where tools have to automatically find invariants) and semi-automated tools (where users have to provide invariants as hints for verification). In particular, finding appropriate qualifiers (sometimes called features [18], and also predicates in verification methods based on predicate abstraction), which are atomic formulas that constitute inductive invariants, has been a challenge. Various machine learning techniques have recently been applied to the discovery of invariants and/or qualifiers. As already mentioned, Garg et al. [6,7] proposed a framework of semi-supervised learning called ICE learning, where implication constraints are provided to a learner in addition to positive and negative examples. The framework has later been generalized for CHC solving $[4,5]$, but the discovery of qualifiers remained as a main bottleneck.

To address the issue of qualifier discovery, Zhu et al. [31] proposed a use of SVMs (support vector machines). Whilst SVMs are typically much faster than

\footnotetext{
18 https://github.com/hopv/benchmarks.
} 
NNs, there are significant shortcomings: (i) SVMs are not good at finding a Boolean combination of linear inequalities (like $A \wedge B$ ) as a classifier. To address the issue, they combined SVMs (to find each qualifier $A, B$ ) with the Boolean decision tree construction [7], but it is in general unlikely that SVMs generate $A$ and/or $B$ as classifiers when $A \wedge B$ is a complete classifier (see Figure 8). (ii) SVMs do not properly take implication constraints into account. Zhu et al. [31] label the data occurring only in implication constraints as positive or negative examples in an ad hoc manner, and pass the labeled data to SVMs. The problem with that approach is that the labeling of data is performed without considering the overall classification problem. Recall the example problem in Section 3.2 consisting of implication constraints of the form $p(2 n) \wedge p(2 n+1) \Rightarrow$ and $\Rightarrow p(2 n) \vee p(2 n+1)$ for $n \in[-10,10]$. In this case, there are $2^{21}$ possible ways to classify data, of which only two classifications $(p(2 n)=$ true and $p(2 n+1)=$ false for all $n$, or $p(2 n+1)=$ true and $p(2 n)=$ false for all $n$ ) lead to a concise classification of $n \bmod 2=0$ or $n \bmod 2=1$. Sharma et al. [25] also applied SVMs to find interpolants. It would be interesting to investigate whether our approach is also useful for finding interpolants.

Padhi et al. $[18,17]$ proposed a method for finding qualifiers (features, in their terminology) based on a technique of syntax-guided program synthesis and combined it with Boolean function learning. Since they enumerate possible features in the increasing order of the size of feature expressions ([18], Figure 5), we think they are not good at finding a feature of large size (like quadratic constraints used in our experiment in Section 2.3). Anyway, since our technique has its own defects (in particular, for finding simple invariants, it is too much slower than the techniques above), our technique should be used as complementary to existing techniques. Si et al. [26] used neural reinforcement learning for learning loop invariants, but in a way quite different from ours. Rather than finding invariants in a data-driven manner, they let NNs to learn invariants from a graph representation of programs.

Although the goal is different, our technique is also related with the NNbased symbolic regression or extrapolation $[15,20,23]$, where the goal is to learn a simple mathematical function expression (like $f(x)=\sin \left(x+x^{2}\right)$ ) from sample pairs of inputs and outputs. To this end, Martius and Lambert's method [15] prepares a $\mathrm{NN}$ whose activation functions are basic mathematical functions (like $\sin$ ), trains it, and extracts the function from the trained NN. The main difference of our approach from theirs is to use NNs as a gray (rather than white) box, only for extracting qualifiers, rather than extracting the whole function computed by NN. Nevertheless, we expect that some of their techniques would be useful also in our context, especially for learning non-linear qualifiers.

\section{Conclusion}

We have proposed a novel method for synthesizing logical formulas using neural networks as gray boxes. Our key insight was that we should be able to extract useful information from trained NNs, if the NNs are suitably designed with 


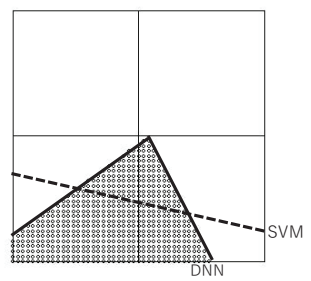

Fig. 8. SVM vs NN: Small circles denote positive examples, and the white space is filled with negative examples. The dashed line shows a linear classifier typically output by a SVM, while the thick line shows the (complete) classifier output by a NN.

the extraction in mind. The results of our preliminary experiments are quite promising. We have also discussed an application of our NN-guided synthesis to program verification through $\mathrm{CHC}$ solving. (Another application to program synthesis through the notion of oracle-based programming is also discussed in Appendix 4. We believe that program verification and synthesis are attractive application domains of neural networks (and machine learning in general), as training data can be automatically collected. We plan to extend our NN-guided synthesis tool to enable the synthesis of (i) functions returning non-Boolean values (such as integers), (ii) predicates/functions on recursive data structures, and (iii) program expressions containing loops. For (ii) and (iii), we plan to deploy recurrent neural networks.

Acknowledgments We would like to thank anonymous referees for useful comments. This work was supported by JSPS KAKENHI Grant Numbers JP20H05703, JP20H04162, and JP19K22842, and ERATO HASUO Metamathematics for Systems Design Project (No. JPMJER1603), JST.

\section{References}

1. Anderson, G., Verma, A., Dillig, I., Chaudhuri, S.: Neurosymbolic reinforcement learning with formally verified exploration. In: Advances in Neural Information Processing Systems 33: Annual Conference on Neural Information Processing Systems 2020, NeurIPS 2020 (2020)

2. Arrieta, A.B., Rodríguez, N.D., Ser, J.D., Bennetot, A., Tabik, S., Barbado, A., García, S., Gil-Lopez, S., Molina, D., Benjamins, R., Chatila, R., Herrera, F.: Explainable artificial intelligence (XAI): concepts, taxonomies, opportunities and challenges toward responsible AI. Inf. Fusion 58, 82-115 (2020). https://doi.org/10.1016/j.inffus.2019.12.012

3. Bjørner, N., Gurfinkel, A., McMillan, K.L., Rybalchenko, A.: Horn clause solvers for program verification. In: Fields of Logic and Computation II - Essays Dedicated to Yuri Gurevich on the Occasion of His 75th Birthday. LNCS, vol. 9300, pp. 24-51. Springer (2015) 
4. Champion, A., Chiba, T., Kobayashi, N., Sato, R.: ICE-based refinement type discovery for higher-order functional programs. J. Autom. Reason. 64(7), 13931418 (2020), https://doi.org/10.1007/s10817-020-09571-y

5. Ezudheen, P., Neider, D., D'Souza, D., Garg, P., Madhusudan, P.: Horn-ICE learning for synthesizing invariants and contracts. Proc. ACM Program. Lang. 2(OOPSLA), 131:1-131:25 (2018), https://doi.org/10.1145/3276501

6. Garg, P., Löding, C., Madhusudan, P., Neider, D.: ICE: A robust framework for learning invariants. In: Proceedings of CAV 2014. LNCS, vol. 8559, pp. 69-87. Springer (2014)

7. Garg, P., Neider, D., Madhusudan, P., Roth, D.: Learning invariants using decision trees and implication counterexamples. In: Proceedings of the 43rd Annual ACM SIGPLAN-SIGACT Symposium on Principles of Programming Languages, POPL 2016. pp. 499-512. ACM (2016), https://doi.org/10.1145/2837614.2837664

8. Gehr, T., Mirman, M., Drachsler-Cohen, D., Tsankov, P., Chaudhuri, S., Vechev, M.T.: AI2: safety and robustness certification of neural networks with abstract interpretation. In: 2018 IEEE Symposium on Security and Privacy, SP 2018, Proceedings. pp. 3-18. IEEE Computer Society (2018), https://doi.org/10.1109/SP. 2018.00058

9. Hojjat, H., Rümmer, P.: The eldarica horn solver. In: 2018 Formal Methods in Computer Aided Design (FMCAD). pp. 1-7 (2018)

10. Huang, X., Kwiatkowska, M., Wang, S., Wu, M.: Safety verification of deep neural networks. In: Computer Aided Verification - 29th International Conference, CAV 2017, Proceedings, Part I. LNCS, vol. 10426, pp. 3-29. Springer (2017). https://doi.org/10.1007/978-3-319-63387-9_1

11. Katz, G., Barrett, C.W., Dill, D.L., Julian, K., Kochenderfer, M.J.: Reluplex: An efficient SMT solver for verifying deep neural networks. In: Computer Aided Verification - 29th International Conference, CAV 2017, Proceedings, Part I. LNCS, vol. 10426, pp. 97-117. Springer (2017), https://doi.org/10.1007/978-3-319-63387-9_5

12. Kingma, D.P., Ba, J.: Adam: A method for stochastic optimization. In: 3rd International Conference on Learning Representations, ICLR 2015, Conference Track Proceedings (2015), http://arxiv.org/abs/1412.6980

13. Kobayashi, N., Sekiyama, T., Sato, I., Unno, H.: Toward neural-network-guided program synthesis and verification. CoRR abs/2103.09414 (2021), https://arxiv. org/abs/2103.09414

14. Komuravelli, A., Gurfinkel, A., Chaki, S.: Smt-based model checking for recursive programs. Formal Methods Syst. Des. 48(3), 175-205 (2016), https://doi.org/10. 1007/s10703-016-0249-4

15. Martius, G., Lampert, C.H.: Extrapolation and learning equations. In: 5th International Conference on Learning Representations, ICLR 2017, Toulon, France, April 24-26, 2017, Workshop Track Proceedings. OpenReview.net (2017), https: //openreview.net/forum?id=BkgRp0FYe

16. Narodytska, N., Kasiviswanathan, S.P., Ryzhyk, L., Sagiv, M., Walsh, T.: Verifying properties of binarized deep neural networks. In: Proceedings of the Thirty-Second AAAI Conference on Artificial Intelligence, (AAAI-18), the 30th innovative Applications of Artificial Intelligence (IAAI-18), and the 8th AAAI Symposium on Educational Advances in Artificial Intelligence (EAAI-18). pp. 6615-6624. AAAI Press (2018)

17. Padhi, S., Millstein, T.D., Nori, A.V., Sharma, R.: Overfitting in synthesis: Theory and practice. In: Computer Aided Verification - 31st International Conference, CAV 2019, Proceedings, Part I. Lecture Notes in Computer Science, vol. 11561, pp. 315-334. Springer (2019). https://doi.org/10.1007/978-3-030-25540-4_17 
18. Padhi, S., Sharma, R., Millstein, T.D.: Data-driven precondition inference with learned features. In: Krintz, C., Berger, E. (eds.) Proceedings of the 37th ACM SIGPLAN Conference on Programming Language Design and Implementation, PLDI 2016, Santa Barbara, CA, USA, June 13-17, 2016. pp. 42-56. ACM (2016), https://doi.org/10.1145/2908080.2908099

19. Pavlinovic, Z., Su, Y., Wies, T.: Data flow refinement type inference. Proc. ACM Program. Lang. 5(POPL), 1-31 (2021). https://doi.org/10.1145/3434300

20. Petersen, B.K., Landajuela, M., Mundhenk, T.N., Santiago, C.P., Kim, S.K., Kim, J.T.: Deep symbolic regression: Recovering mathematical expressions from data via risk-seeking policy gradients. In: Proceedings of the International Conference on Learning Representations (2021)

21. Pulina, L., Tacchella, A.: An abstraction-refinement approach to verification of artificial neural networks. In: Computer Aided Verification, 22nd International Conference, CAV 2010, Proceedings. LNCS, vol. 6174, pp. 243-257. Springer (2010). https://doi.org/10.1007/978-3-642-14295-6_24

22. Ryan, G., Wong, J., Yao, J., Gu, R., Jana, S.: CLN2INV: learning loop invariants with continuous logic networks. In: 8th International Conference on Learning Representations, ICLR 2020. OpenReview.net (2020)

23. Sahoo, S., Lampert, C., Martius, G.: Learning equations for extrapolation and control. In: Dy, J., Krause, A. (eds.) Proceedings of the 35th International Conference on Machine Learning. Proceedings of Machine Learning Research, vol. 80, pp. 4442-4450. PMLR, Stockholmsmässan, Stockholm Sweden (10-15 Jul 2018), http://proceedings.mlr.press/v80/sahoo18a.html

24. Satake, Y., Unno, H., Yanagi, H.: Probabilistic inference for predicate constraint satisfaction. In: The Thirty-Fourth AAAI Conference on Artificial Intelligence, AAAI 2020, The Thirty-Second Innovative Applications of Artificial Intelligence Conference, IAAI 2020, The Tenth AAAI Symposium on Educational Advances in Artificial Intelligence, EAAI 2020. pp. 1644-1651. AAAI Press (2020)

25. Sharma, R., Nori, A.V., Aiken, A.: Interpolants as classifiers. In: Madhusudan, P., Seshia, S.A. (eds.) Computer Aided Verification - 24th International Conference, CAV 2012, Proceedings. LNCS, vol. 7358, pp. 71-87. Springer (2012). https://doi.org/10.1007/978-3-642-31424-7_11

26. Si, X., Dai, H., Raghothaman, M., Naik, M., Song, L.: Learning loop invariants for program verification. In: Advances in Neural Information Processing Systems 31: Annual Conference on Neural Information Processing Systems 2018, NeurIPS 2018. pp. 7762-7773 (2018)

27. Solar-Lezama, A.: Program synthesis by sketching. Ph.D. thesis, University of California, Berkeley (2008)

28. Verma, A., Murali, V., Singh, R., Kohli, P., Chaudhuri, S.: Programmatically interpretable reinforcement learning. In: Proceedings of the 35th International Conference on Machine Learning, ICML 2018. Proceedings of Machine Learning Research, vol. 80, pp. 5052-5061. PMLR (2018)

29. Yao, J., Ryan, G., Wong, J., Jana, S., Gu, R.: Learning nonlinear loop invariants with gated continuous logic networks. In: Proceedings of the 41st ACM SIGPLAN International Conference on Programming Language Design and Implementation, PLDI 2020. pp. 106-120. ACM (2020). https://doi.org/10.1145/3385412.3385986

30. Zhao, H., Zeng, X., Chen, T., Liu, Z., Woodcock, J.: Learning safe neural network controllers with barrier certificates (2020)

31. Zhu, H., Magill, S., Jagannathan, S.: A data-driven CHC solver. In: Proceedings of the 39th ACM SIGPLAN Conference on Programming Lan- 
guage Design and Implementation, PLDI 2018. pp. 707-721. ACM (2018). https://doi.org/10.1145/3192366.3192416

32. Zhu, H., Xiong, Z., Magill, S., Jagannathan, S.: An inductive synthesis framework for verifiable reinforcement learning. In: Proceedings of the 40th ACM SIGPLAN Conference on Programming Language Design and Implementation, PLDI 2019. pp. 686-701. ACM (2019). https://doi.org/10.1145/3314221.3314638

\section{Appendix}

\section{A Additional Information for Section 2}

\section{A.1 Instances for Learning $(A \wedge B) \vee(C \wedge D)$}

Below is the visualization of all the 20 instances used in the experiments for learning formulas of the form $(A \wedge B) \vee(C \wedge D)$. The small circles represent positive examples, and the white area is (implicitly) filled with negative examples.

Instance \#1:

$(x-4 y-9>0 \wedge 2 x-y-9>0)$

$\vee(-x+y-1>0 \wedge x+2 y+2>0)$

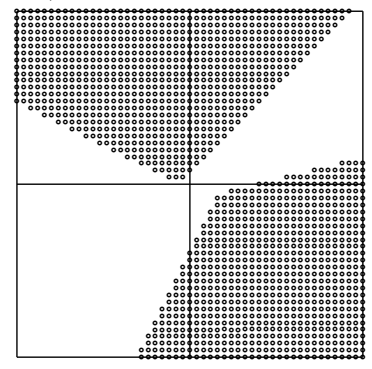

Instance \#3:

$(x+2 y-5>0 \wedge-x+y>0)$

$\vee(-4 x+y>0 \wedge x-4 y+5>0)$

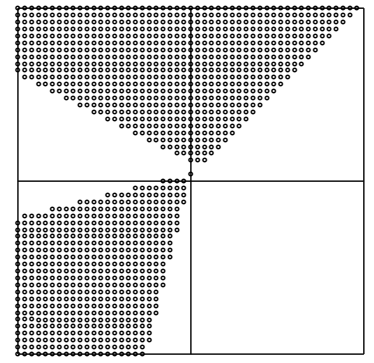

Instance \#2:

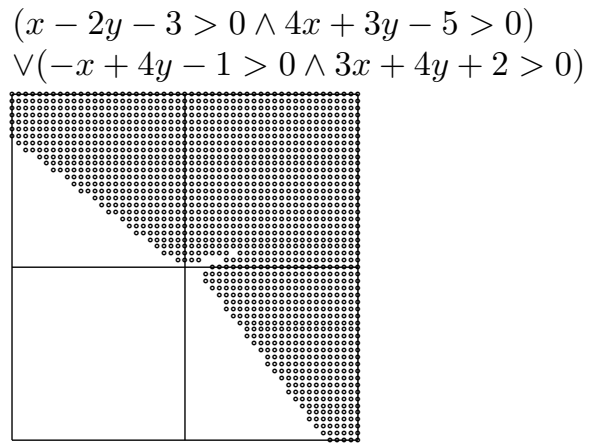

Instance \#4:

$(x-2 y-6>0 \wedge-3 x-2 y+4>0)$

$\vee(-4 x+y-5>0 \wedge-3 x+4 y-1>0)$

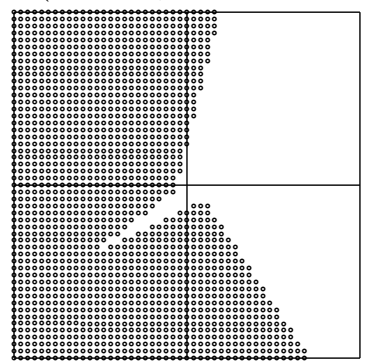


Instance \#5:

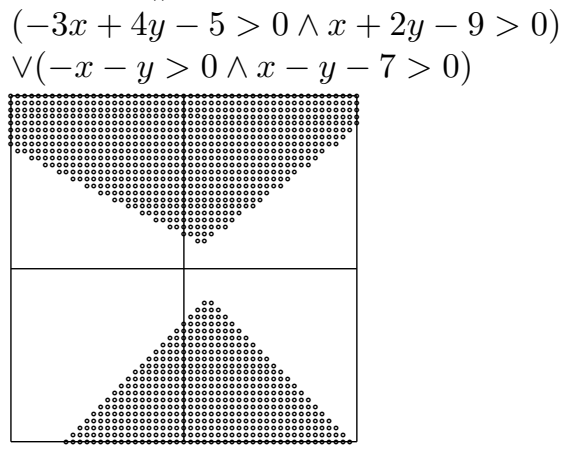

Instance \#7:

$(2 x+y+2>0 \wedge x-y+2>0)$

$\vee(-x-2 y-7>0 \wedge-x+2 y+3>0)$

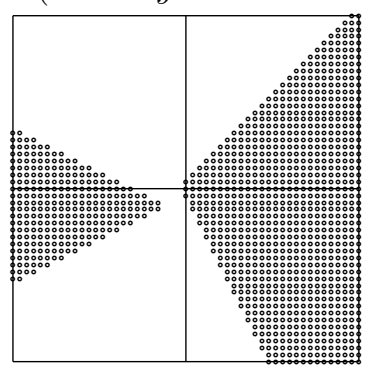

Instance \#9:

$(x+4 y-4>0 \wedge 2 x+y+1>0)$

$\vee(-x-y+4>0 \wedge 4 x-y+5>0)$

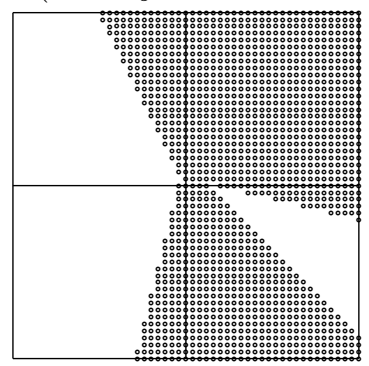

Instance \#6:

$(2 x-y-9>0 \wedge x+4 y-5>0)$

$\vee(-3 x+2 y+7>0 \wedge-2 x-3 y-3>0)$

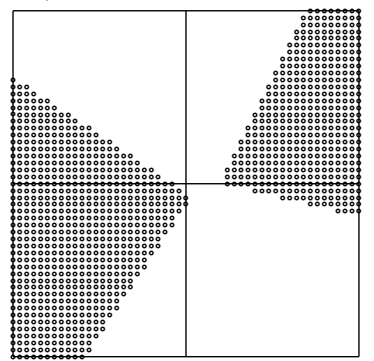

Instance \#8:

$(-x+3 y-1>0 \wedge-4 x+3 y-2>0)$

$\vee(-4 x-3 y-2>0 \wedge 3 x-4 y+7>0)$

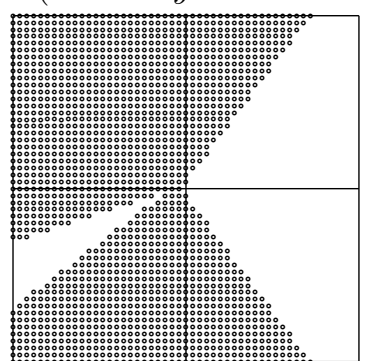

Instance \#10:

$(2 x+y+1>0 \wedge x-y-9>0)$

$\vee(x+y>0 \wedge 3 x-y-5>0)$

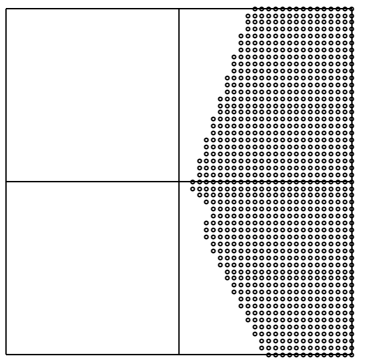


Instance \#11

$(2 x+y>0 \wedge-3 x+2 y+8>0)$ $\vee(4 x-y+5>0 \wedge x-2 y-6>0)$

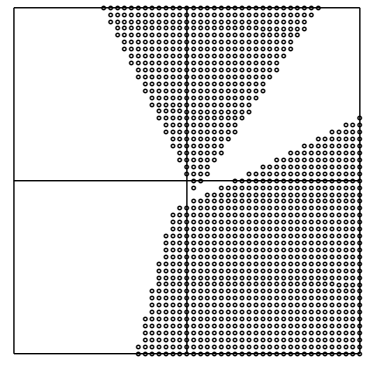

Instance \#13:

$(-2 x+3 y+9>0 \wedge-x-y+1>0)$

$\vee(3 x+4 y-9>0 \wedge-2 x+y+4>0)$

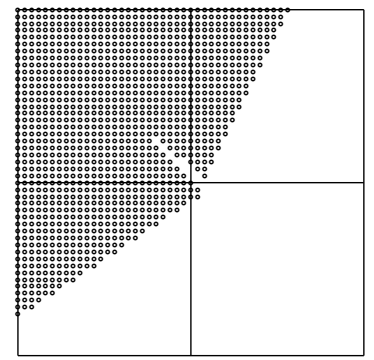

Instance \#15:

$(4 x-3 y-7>0 \wedge 2 x+3 y+7>0)$

$\vee(-x+y+4>0 \wedge-x-2 y-2>0)$

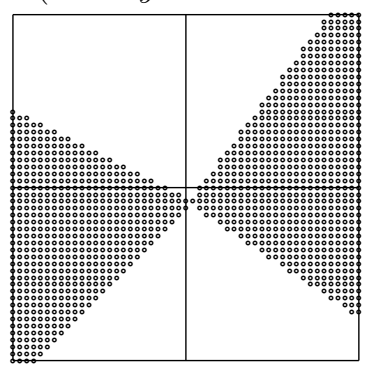

Instance \#12:

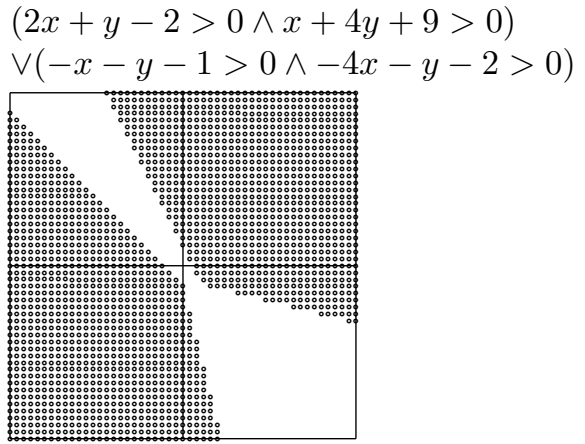

Instance \#14:

$(3 x+y+6>0 \wedge-3 x-4 y+1>0)$

$\vee(-3 x+2 y+9>0 \wedge x+y-9>0)$

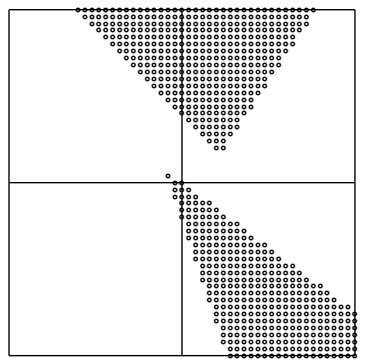

Instance \#16:

$(4 x+3 y-4>0 \wedge-4 x+y+9>0)$ $\vee(-2 x+y-1>0 \wedge-x-4 y-2>0)$

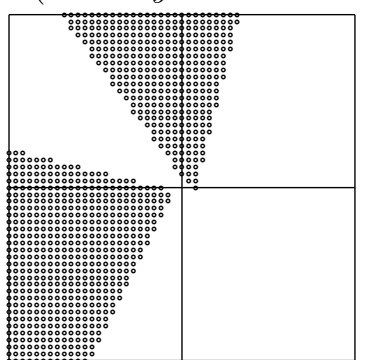


Instance \#17:

$(-4 x-y+1>0 \wedge 3 x-4 y-8>0)$

$\vee(2 x+3 y+6>0 \wedge 3 x-4 y-4>0)$

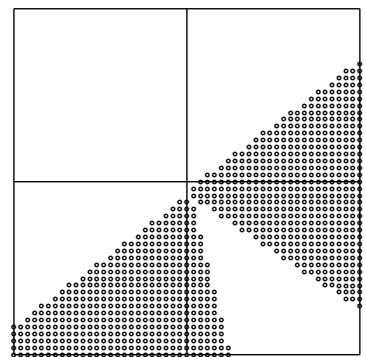

Instance \#19:

$(-4 x-3 y-2>0 \wedge x-2 y-9>0)$

$\vee(x+2 y>0 \wedge 3 x-y-6>0)$

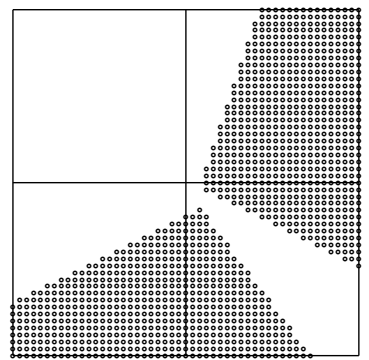

Instance \#18:

$(x-y>0 \wedge-2 x-y-7>0)$

$\vee(-2 x+y-7>0 \wedge x+y+1>0)$

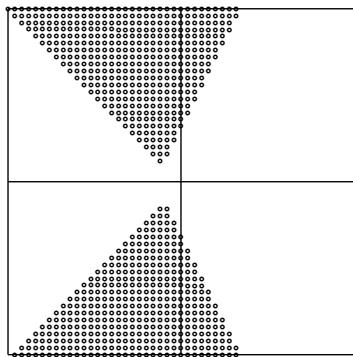

Instance \#20:

$(-x-y+2>0 \wedge 2 x-y-3>0)$

$\vee(x+2 y+2>0 \wedge-x+2 y+7>0)$

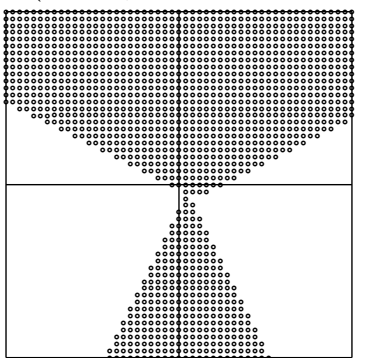

\section{A.2 Instances for Quadratic Constraints}

Instance \#1:

$-x^{2}+3 x y-3 y^{2}-x+y+89>0$

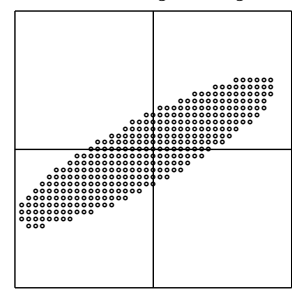

Instance \#3:

$x^{2}+x y+2 y^{2}+2 x-2 y-179>0$

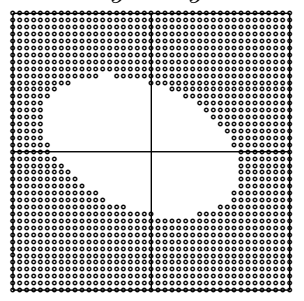

Instance \#2:

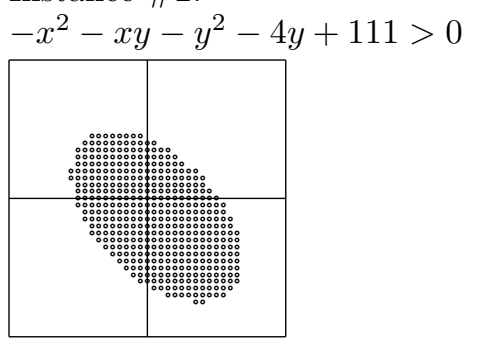

Instance \#4:

$-3 x^{2}+2 x y-y^{2}+2 x-3 y+154>0$

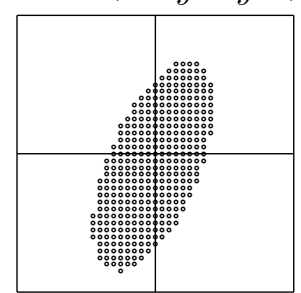


Instance \#5:

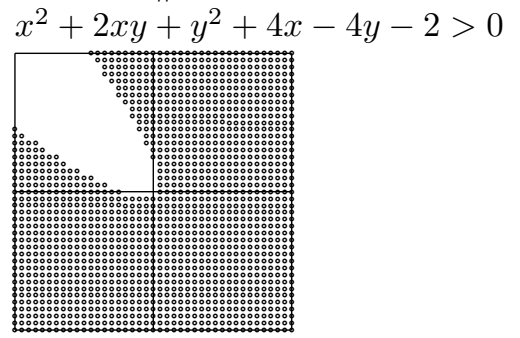

Instance \#7:

$-x^{2}-2 x y-y^{2}+4 x-4 y+5>0$

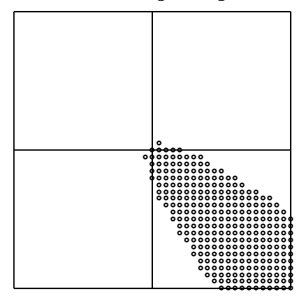

Instance \#9:

$2 x^{2}-y^{2}+x+2 y-6>0$

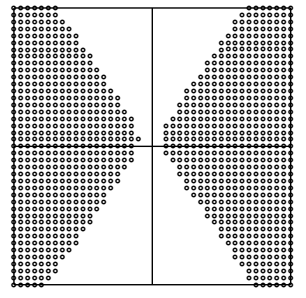

Instance \#11:

$x^{2}+2 x y-x+4>0$

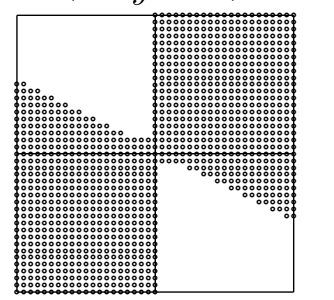

Instance \#6:

$x^{2}-2 x y+y^{2}+4 x+2 y-9>0$

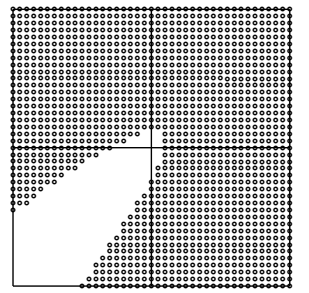

Instance \#8:

$x^{2}+3 x-4 y-6>0$

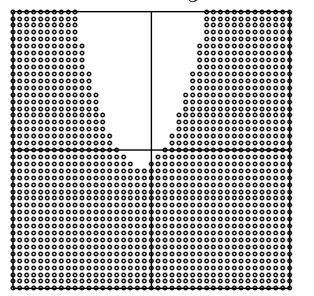

Instance \#10:

$-x^{2}+4 x y-y^{2}-4 x-9>0$

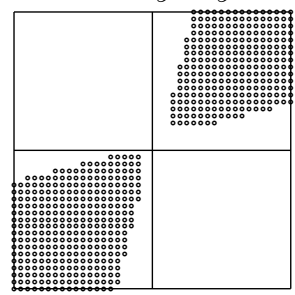

Instance \#12:

$x^{2}-3 x y-4 y^{2}-4 x-3 y-4>0$

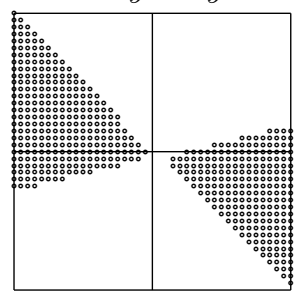


Instance \#13:

Instance \#14:

$-3 x^{2}+3 x y-y^{2}-2 x+158>0 \quad-4 x^{2}+3 x y+2 y^{2}-4 x+4 y+2>0$

$\vee-2 x^{2}-4 x y-3 y^{2}+4 x+3 y+174>0 \vee 2 x^{2}-2 x y-4 y^{2}-x+3 y+5>0$

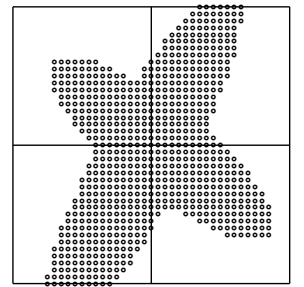

Instance \#15:

$-x^{2}-4 x+3 y+9>0$

$\vee-x^{2}-2 x y-y^{2}+4 x-4 y+8>0$

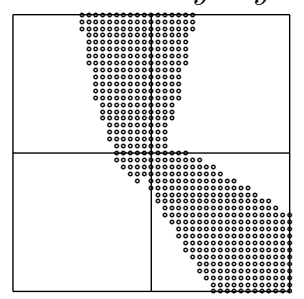

Instance \#17:

$-x^{2}-3 x+4 y+9>0$

$\vee x^{2}+x y-2 y^{2}+3 x-8>0$

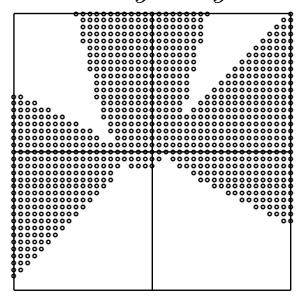

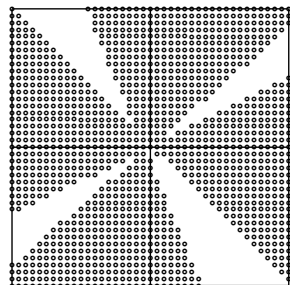

Instance \#16:

$-x^{2}+4 x+4 y+8>0$

$\vee-2 x^{2}-2 x y-2 y^{2}-3 x-4 y+199>0$

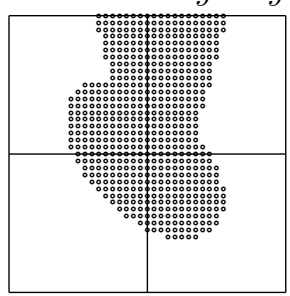

Instance \#18:

$-2 x^{2}+x y-y^{2}+4 x-y+160>0$

$\vee-4 x^{2}-4 x y+y^{2}+2 x-2 y-2>0$

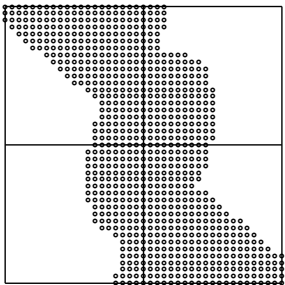

\section{A.3 On Three-Layer vs Four-Layer NNs}

As reported in Section 2.2, three-layer NNs with a sufficient number of nodes performed well in the experiment on learning formulas $(A \wedge B) \vee(C \wedge D)$, contrary to our expectation. This section reports our analysis to find out the reason.

We used the instance shown on the left-hand side of Figure 9, and compared the training results of three-layer and four-layer NNs. To make the analysis easier, we tried to train the NNs with a minimal number of hidden nodes in the second layer. For the four-layer-case, the training succeeded for only two hidden nodes in the second layer (plus eight hidden nodes in the third layer), and only relevant quantifiers of the form $x>c, x<c, y>c, y<c$ for $c \in\{-2,1,0,1\}$ were generated. In contrast, for the three-layer case, 12 hidden nodes were required for the training to succeed. The right-hand side of Figure 9 shows the lines $b_{i}+w_{i, x} x+w_{i, y} y=0(i \in\{1, \ldots, 12\})$ where $w_{i, x}, w_{i, y}$ and $b_{i}$ are the weights 
and bias for the $i$-th hidden node. We can see that the lines that are (seemingly) irrelevant to the original formula are recognized by the hidden nodes. Removing any hidden node (e.g., the node corresponding to line 1) makes the NN fail to properly separate positive and negative examples. Thus, the three-layer NN is recognizing positive and negative examples in a manner quite different from the original formula; it is performing a kind of patchwork to classify the examples. Nevertheless, even in that patchwork, there are lines close to the horizontal and vertical lines $y=0$ and $x=0$. Thus, if we use the trained NN only to extract qualifier candidates (rather than to recover the whole formula by inspecting also the weights of the third layer), three-layer NNs can be useful, as already observed in the experiments in Section 2.2.
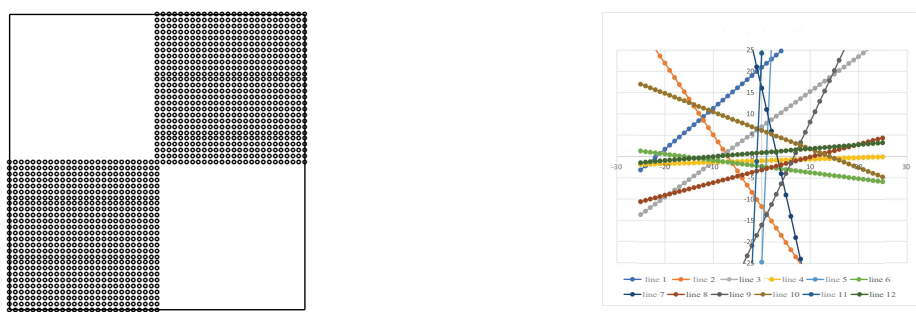

Fig. 9. Problem Instance for Learning $(x \geq 0 \wedge y \geq 0) \vee(x \leq 0 \wedge y \leq 0)$ (left) and Lines Recognized by Three-Layer NNs for Problem Instance $(x \geq 0 \wedge y \geq 0) \vee(x \leq 0 \wedge y \leq 0)$ (right).

\section{A.4 On Activation Functions}

To justify our choice of the sigmoid function as activation functions, we have replaced the activation functions with $\operatorname{ReLU}(f(x)=x$ for $x \geq 0$ and $f(x)=0$ for $x<0)$ and Leaky ReLU $(f(x)=x$ for $x \geq 0$ and $f(x)=-0.01 x$ for $x<0)$ and conducted the experiments for synthesizing $(A \wedge B) \vee(C \wedge D)$ by using the same problem instances as those used in Section 2.2. Here are the results.

\begin{tabular}{|c|c|c|c|c|c|c|}
\hline activation & \#hidden nodes & \#retry & \%success & \% qualifiers & \#candidates & time (sec.) \\
\hline \hline ReLU & $32: 32$ & 15 & $18.3 \%$ & $66.7 \%$ & 72.4 & 55.9 \\
\hline Leaky ReLU & $32: 32$ & 32 & $61.7 \%$ & $88.8 \%$ & 64.4 & 52.8 \\
\hline sigmoid & $32: 32$ & 10 & $85.0 \%$ & $91.7 \%$ & 40.4 & 39.9 \\
\hline
\end{tabular}

In this experiment, we have used the mean square error function as the loss function (since the log loss function assumes that the output belongs to $[0,1]$, which is not the case here). For comparison, we have re-shown the result for the sigmoid function (with the mean square error loss function).

As clear from the table above, the sigmoid function performs significantly better than ReLU and Leaky ReLU, especially in \%success (the larger is better) 
and \#candidates (the smaller is better). This confirms our expectation that the use of the sigmoid function helps us to ensure that only information about $b+w_{1} x_{1}+\cdots+w_{k} x_{k}>c$ for small $c$ 's may be propagated to the output of the second layer, so that we can find suitable qualifiers by looking at only the weights and biases for the hidden nodes in the second layer. We do not report experimental results for the tanh function $(\tanh (x)=2 \sigma(x)-1)$, but it should be as good as the sigmoid function, as it has similar characteristics.

\section{A.5 On Biases}

As for the biases in the second later, we have actually removed them and instead added a constant input 1, so that the weights for the constant input play the role of the biases (thus, for two-dimensional input $(x, y)$, we actually gave threedimensional input $(x, y, 1))$. This is because, for some qualifier that requires a large constant (like $x+y-150>0$ ), adding additional constant inputs such as 100 (so that inputs are now of the form $(x, y, 1,100)$ ) makes the NN training easier to succeed. Among the experiments reported in this paper, we added an additional constant input 10 for the experiments in Section 2.3.

Similarly, we also observed (in the experiments not reported here) that, when the scales of inputs vary extremely among different dimensions (like $(x, y)=$ $(1,100),(2,200), \ldots)$, then the normalization of the inputs helped the convergence of training.

\section{B Additional Information for Section 3}

Here we provide more details about our experiments on the CHC problem xyz, which shows a general pitfall of the ICE-based CHC solving approach of HoICE (rather than that of our neural network-guided approach). Here is the source program of xyz written in OCaml (we have simplified the original program, by removing redundant arguments).

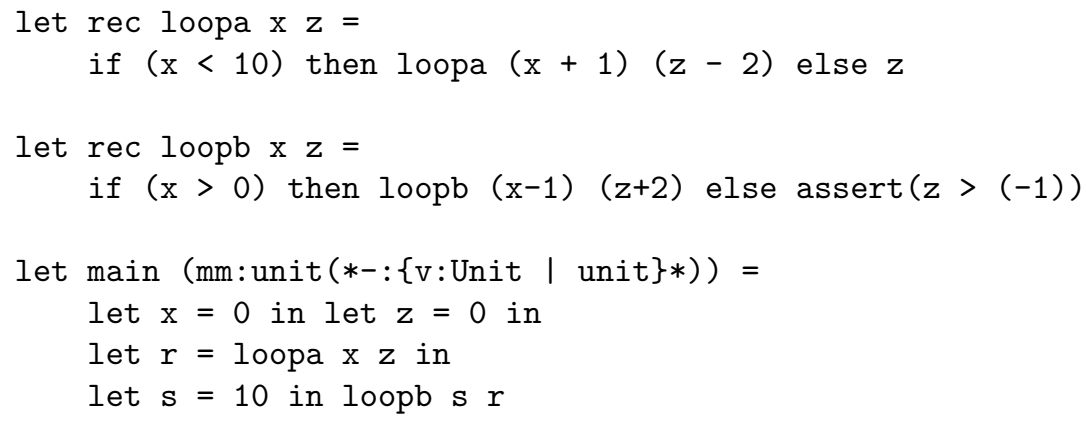

Here is (a simplified version of) the corresponding CHC generated by r_type.

$$
\begin{aligned}
x<10 \wedge \operatorname{loopa}(x+1, z-2, r) & \Rightarrow \operatorname{loopa}(x, z, r) \\
x \geq 10 & \Rightarrow \operatorname{loopa}(x, z, z)
\end{aligned}
$$




$$
\begin{aligned}
x>0 \wedge \operatorname{loopb}(x, z) & \Rightarrow \operatorname{loopb}(x-1, z+2) \\
x \leq 0 \wedge \operatorname{loopb}(x, z) & \Rightarrow z>-1 \\
\operatorname{loopa}(0,0, r) & \Rightarrow \operatorname{loopb}(10, r)
\end{aligned}
$$

When we ran HoIcE for collecting learning data, we observed that no positive examples for loopb and no negative examples for loopa were collected. Thus NeuGuS returns a trivial solution such as loopa $(x, z, r) \equiv$ true and loop $b(x, z) \equiv$ false. The reason why HoICE generates no positive examples for loopb is as follows. A positive example of loopb can only be generated from the clause (5), only when a positive example of the form loopa $(0,0, r)$ is already available. To generate a positive example of the form loopa $(0,0, r)$, however, one needs to properly instantiate the clauses (2) and (1) repeatedly; since HoICE generates examples only lazily when a candidate model returned by the learner does not satisfy the clauses. In short, HoICE must follow a very narrow sequence of non-deterministic choices to generate the first positive example of loopb. Negative examples of loopa are rarely generated for the same reason.

Another obstacle is that even if HoICE can generate a negative counterexample through clause (5) with a luck, it is only of the form loopa $(0,0, r)$. Although further negative examples can be generated through (1), the shape of the resulting negative examples are quite limited.

To deal with the problem above, we have prepared an option to collect learning data also directly from $\mathrm{CHCs}$, by unit propagation. For example, the negative example $\operatorname{loop} b(0,-1) \Rightarrow$ false can be generated from the clause 4 above. By combining it with the clause 3 , negative examples loopb $(1,-3) \Rightarrow$ $, \ldots, \operatorname{loop} b(10,-21) \Rightarrow$ are generated. By combining $\operatorname{loop} b(10,-21) \Rightarrow$ with the clause 5 , the negative example loopa $(0,0,-21) \Rightarrow$ can be generated. As reported in Section 3, with this option enabled, our tool could successfully solve xyz.

The variant $x_{y} z_{-} v$ comes from the following variation of the OCaml program, obtained by generalizing 10 and 0 to arbitrary integer values. Thus, from the viewpoint of program verification, the variant is harder to verify: in fact, Z3 times-out for this variant. Our tool, however, actually runs faster for the variant, because negative examples are easier to collect for this variant.

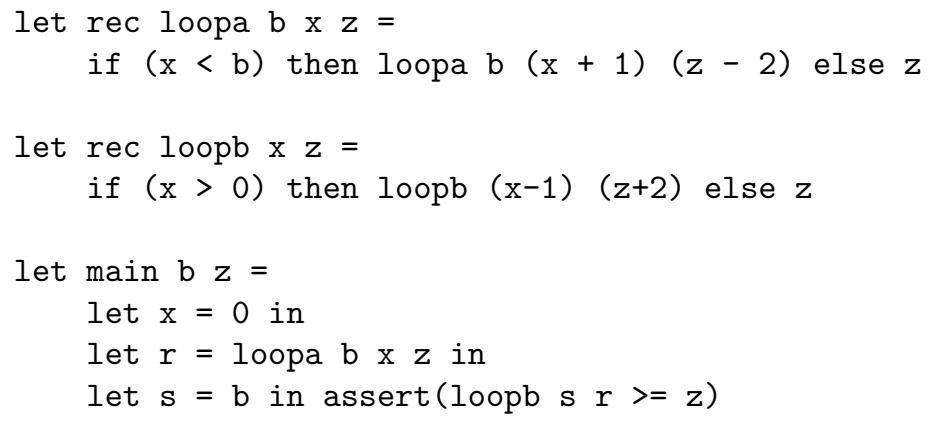




\section{Preliminary Experiment on Programming with Oracles}

We have picked several OCaml programs from the benchmark set in https: //github.com/hopv/benchmarks, and tested our framework. Some of them are reported below.

Consider the following program.

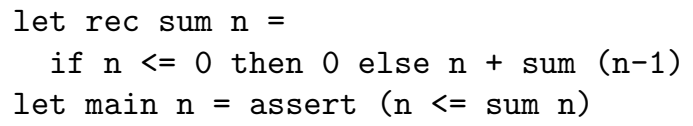

We have replaced the conditional expression with an oracle, and obtained:

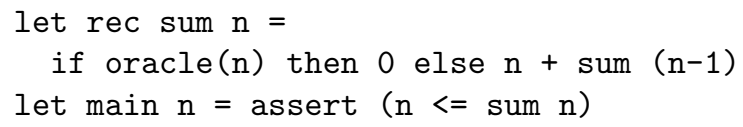

The goal is now to check whether the original expression for oracle(n) (i.e., $\mathrm{n}<=0$ ) can be recovered.

To generate training data for oracle, we used random testing. For that purpose, the code above was surrounded with the following code.

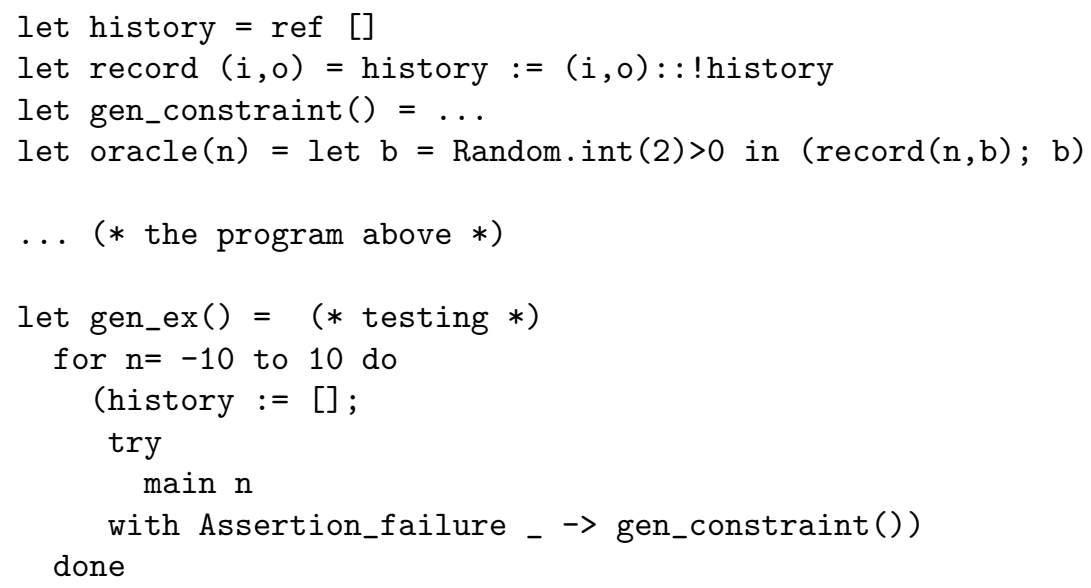

The oracle generates a random Boolean $b$, records it in the call history, and returns $b$. Upon an assertion failure, gen_constraint () is called, which generates implication constraints. By running gen_ex () twice, we obtained the following 
examples.

Negative examples:

oracle(1), oracle(3), oracle(5), oracle(6), oracle(7), oracle(8), oracle(9), oracle $(10)$

Implication examples:

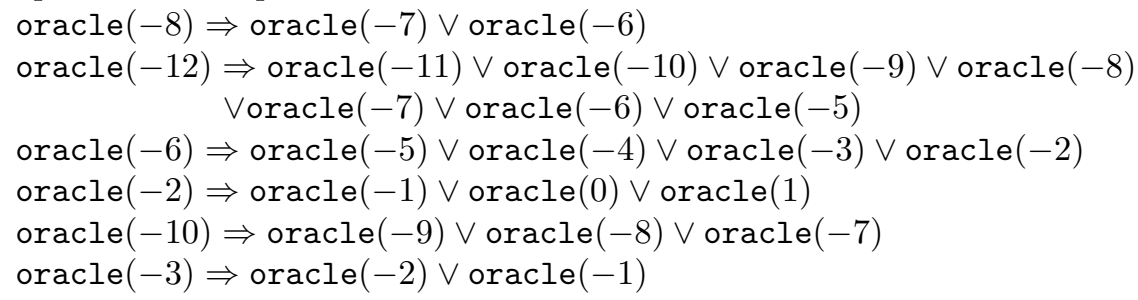

Given the examples above, our synthesis tool returned oracle $(n) \equiv$ false. (Note that it indeed satisfies all the constraints above.)

Then, the resulting program is:

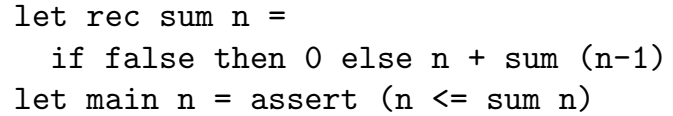

Obviously, the program does not fail, but never terminates. By testing the program above, we obtain an infinite sequence of oracle calls, like:

$$
\operatorname{oracle}(0)=\text { false; } \operatorname{oracle}(-1)=\text { false; oracle }(-2)=\text { false; } \cdots
$$

We have cut the infinite sequence, and added the following implication example:

$$
\Rightarrow \operatorname{oracle}(0) \vee \operatorname{oracle}(-1) \vee \operatorname{oracle}(-2) \vee \cdots \vee \operatorname{oracle}(-5) \text {. }
$$

By running our synthesis tool again, we have obtained oracle $(n) \equiv n<1$. Thus, we obtained

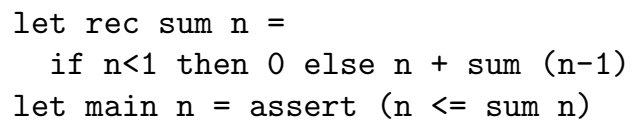

and the resulting program could be verified by MoCHi (https://github.com/ hopv/MoCHi), an automated program verification tool for functional programs.

Let us consider another example. The following program has also been taken from the same benchmark set, where the original conditional expression $x \geq y$ in $f$ has been replaced by an oracle call. we have also replaced the specification as the original assertion $\mathrm{f} \times \mathrm{m}=\mathrm{m}$ admits a silly solution oracle $(x, y)=$ false.

let $\max \max 2(\mathrm{x}:$ int $)(\mathrm{y}:$ int $)(\mathrm{z}:$ int $):$ int $=\max 2(\max 2 \mathrm{x} y) \mathrm{z}$

let $f \mathrm{x} y$ : int $=$ if $\operatorname{oracle}(\mathrm{x}, \mathrm{y})$ then $\mathrm{x}$ else $\mathrm{y}$ 


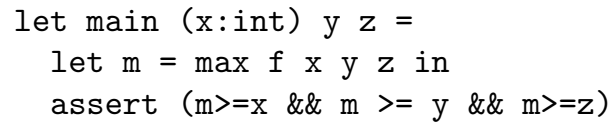

As before, the above code was surrounded with the code for testing the code and generating implication examples. After running the test several times, the following implication examples were accumulated.

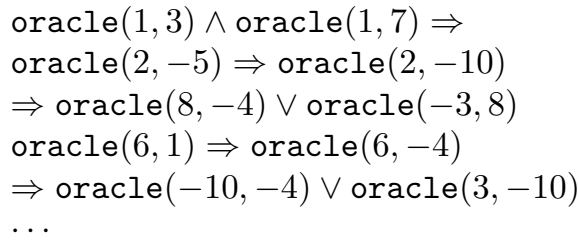

Given those examples, our synthesis tool generated oracle $(x, y) \equiv 2 x-3 y-1>$ 0 , which is a wrong solution. By testing the program obtained by inserting the wrong oracle:

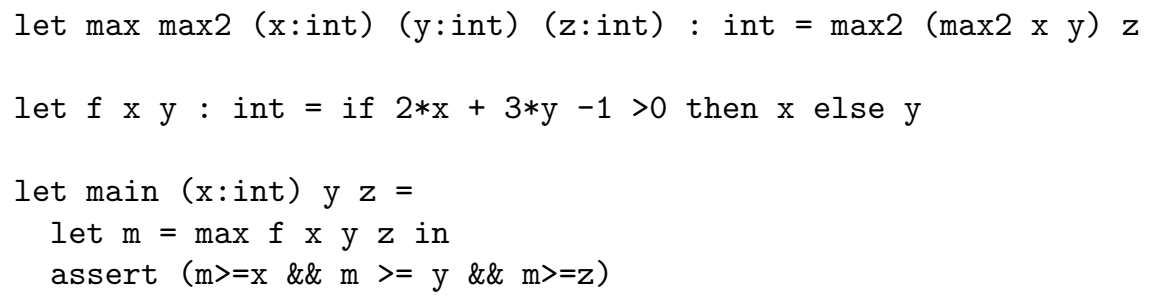

we obtained further implication examples:

$$
\begin{aligned}
& \operatorname{oracle}(9,-6) \Rightarrow \operatorname{oracle}(9,8) \\
& \operatorname{oracle}(8,0) \Rightarrow \operatorname{oracle}(8,6) .
\end{aligned}
$$

After adding them to the training data, our tool generated oracle $(x, y) \equiv x-$ $y+1>0$, which is correct. The resulting program could be verified by MoCHi.

As a little more non-trivial example, we have also tested McCarthy's 91 function:

let $\operatorname{rec} \operatorname{mc91} \mathrm{x}=$ if $\operatorname{oracle}(\mathrm{x})$ then $\mathrm{x}-10$ else mc91 (mc91 $(\mathrm{x}+11))$

let main $\mathrm{n}=$ if $\mathrm{n}<=101$ then assert (mc91 $\mathrm{n}=91)$

As before, we have first replaced oracle(x) with a non-deterministic Boolean function, and collected training data by randomly executing the program. From runs that lead to assertion failures, we could obtain negative examples of the form $\operatorname{oracle}(n) \Rightarrow$ for $0 \leq n \leq 100$ (besides other implication examples). In addition, we have collected positive examples from successful runs that contain only a single oracle call. Actually, the only positive example collected was oracle(101). From those data, our tool could correctly synthesize the formula $x>100$. 